\title{
Finite Correlation Length Scaling in Lorentz-Invariant Gapless iPEPS Wave Functions
}

\author{
Michael Rader and Andreas M. Läuchli \\ Institut für Theoretische Physik, Universität Innsbruck, A-6020 Innsbruck, Austria
}

(Received 28 March 2018; published 30 July 2018)

\begin{abstract}
It is an open question how well tensor network states in the form of an infinite projected entangled-pair states (iPEPS) tensor network can approximate gapless quantum states of matter. Here we address this issue for two different physical scenarios: (i) a conformally invariant $(2+1) d$ quantum critical point in the incarnation of the transverse-field Ising model on the square lattice and (ii) spontaneously broken continuous symmetries with gapless Goldstone modes exemplified by the $S=1 / 2$ antiferromagnetic Heisenberg and $X Y$ models on the square lattice. We find that the energetically best wave functions display finite correlation lengths and we introduce a powerful finite correlation length scaling framework for the analysis of such finite bond dimension (finite- $D$ ) iPEPS states. The framework is important (i) to understand the mild limitations of the finite- $D$ iPEPS manifold in representing Lorentz-invariant, gapless many-body quantum states and (ii) to put forward a practical scheme in which the finite correlation length $\xi(D)$ combined with field theory inspired formulas can be used to extrapolate the data to infinite correlation length, i.e., to the thermodynamic limit. The finite correlation length scaling framework opens the way for further exploration of quantum matter with an (expected) Lorentz-invariant, massless low-energy description, with many applications ranging from condensed matter to high-energy physics.
\end{abstract}

DOI: 10.1103/PhysRevX.8.031030

\section{INTRODUCTION}

The study of interacting quantum matter is of enormous current interest, with questions ranging from quantum spin liquids, topological matter, correlated electrons in solids, and ultracold atoms in optical lattices to strongly coupled quantum field theories.

In this context numerical approaches play a very important role, with tensor networks being a central player. For problems in one spatial dimension, computational methods, such as the density matrix renormalization group [1,2], (infinite) matrix product states [(i)MPS] [3-5] and the multiscale entanglement renormalization ansatz [6] have proven to be very powerful, both for gapped states and for quantum critical states with a low-energy conformal field theory (CFT) description [7-10].

In two spatial dimensions, infinite projected entangledpair states (iPEPS) [11] have become a competitive numerical approach with successful applications to many problems in the field of quantum magnetism and for strongly correlated fermions [12-17]. Furthermore,

Published by the American Physical Society under the terms of the Creative Commons Attribution 4.0 International license. Further distribution of this work must maintain attribution to the author(s) and the published article's title, journal citation, and DOI.

\author{
Subject Areas: Computational Physics, \\ Condensed Matter Physics, \\ Statistical Physics
}

theoretical work has established how different forms of topological order can be represented and understood in iPEPS wave functions with finite bond dimension $[18,19]$.

It is, however, an open question how well tensor network states in the form of an iPEPS tensor network can approximate gapless quantum states of matter. Here we explore this issue for two distinct physical scenarios: (i) a conformally invariant $(2+1) d$ quantum critical point in the incarnation of the transverse-field Ising model on the square lattice and (ii) spontaneously broken continuous symmetries with gapless Goldstone modes exemplified by the $S=1 / 2$ antiferromagnetic Heisenberg and $X Y$ models on the square lattice. We find that the best variational wave functions display finite correlation lengths and we introduce a powerful finite correlation length scaling framework for the analysis of such finite- $D$ iPEPS states.

The outline of this paper is as follows. We start by providing a short introduction to the iPEPS framework and the energy optimization strategies in Sec. II. In Sec. III, we study the critical properties of the $(2+1) d$ transversefield Ising model as an example of a quantum critical point in the $(2+1) d$ Ising universality class. In Sec. IV, we present results for the $S=1 / 2$ antiferromagnetic Heisenberg model and the $S=1 / 2 X Y$ model, as examples for continuous symmetry breaking. In Sec. V, we provide an extensive discussion and interpretation of the results obtained, and we conclude in Sec. VI. 


\section{INFINITE (SYSTEM) PEPS}

Considering a two-dimensional quantum many-body system consisting of $p$-level particles, placed on an infinite square lattice, one can make an ansatz for a wave function describing the system,

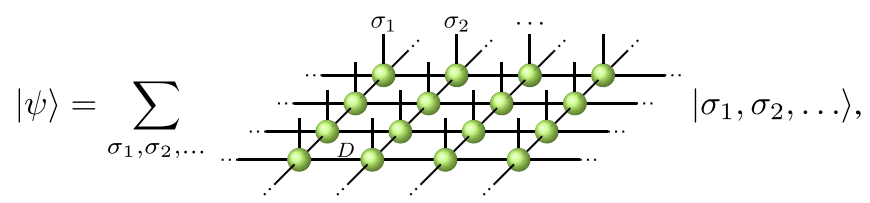

which is commonly known as an infinite system projected entangled-pair state [11].

The graph in Eq. (1) is called a tensor network diagram, where nodes (edges) represent tensors (their corresponding indices). Edges connecting two tensors indicate summation indices and are of dimension $D$, which we call the bond dimension of the iPEPS. The open indices $\sigma_{1}, \sigma_{2}, \ldots$ are of dimension $p$. In this work we consider only iPEPS with a single-site unit cell, i.e., iPEPS where the same tensor is used for each site, but all the techniques described here can be generalized to arbitrary unit cells.

iPEPS are a straightforward generalization of infinite system matrix product states for two spatial dimensions and obey an area law for the entanglement entropy as well $[11,20]$. In contrast to iMPS, iPEPS can be constructed to have infinite correlation lengths, even for the simplest nontrivial case, $D=2$ [21].

\section{A. Contraction}

The key challenge in all iPEPS algorithms is the socalled contraction of the state. For instance, to evaluate single-site observables, one needs the single-site density matrix,

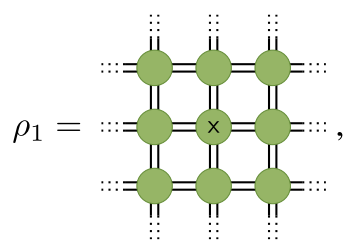

which consists of an infinite sum of double-layer tensors:

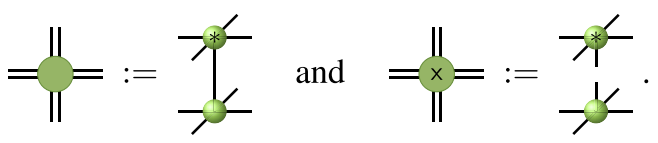

The idea of a contraction is to find an approximation with controllable error for this infinitely large tensor network. There are several ways to go: finding an approximate environment in the form of a boundary matrix product state (bMPS) [22] or a corner transfer matrix (CTM) environment
$[23,24]$ or by directly applying renormalization group (RG) schemes such as tensor RG [25], tensor-entanglement RG [26], second RG [27], higher-order tensor RG [28], or tensor network renormalization [29].

Formally, a bMPS is nothing but an iMPS, with bond dimension $\chi$, that is an approximation for the dominant eigenvector of a transfer matrix of double-layer iPEPS tensors,

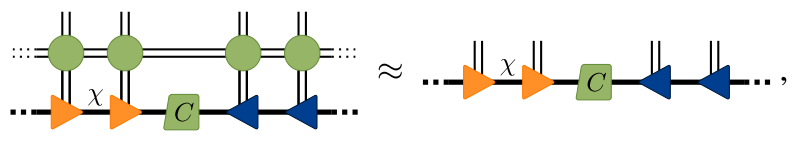

with corresponding eigenvalue 1 . Note that the quality of this approximation can be improved by increasing $\chi$. The triangular tensors in Eq. (4) are isometric, i.e.,

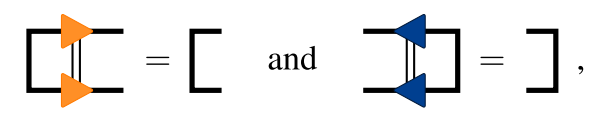

so the bMPS is in a mixed canonical form. From the singular values $\zeta_{j}$ of the central bond matrix $C$, (Rényi) entropies,

$$
S_{\mathrm{bMPS}}^{(\alpha)}=\frac{1}{1-\alpha} \log \left(\sum_{j} \zeta_{j}^{\alpha}\right)
$$

can be computed. In addition to the bMPS tensors, one has to determine a horizontal dominant eigenvector,

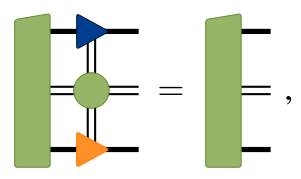

with corresponding eigenvalue 1 to be able to write the single-site density matrix as

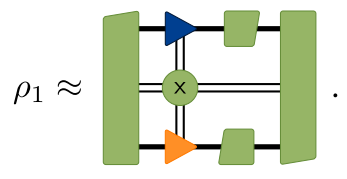

The state-of-the-art method to find such a bMPS is described in Ref. [30] and has also been used in this work.

Another way to contract a state is to find corner transfer matrices,

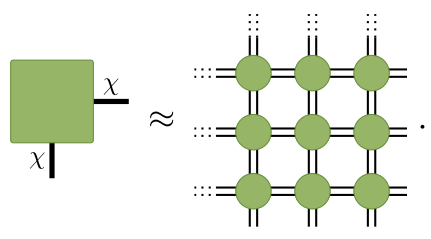


and half-line transfer tensors (HLTTs),

$$
\frac{\chi \mid}{\chi \|}=\approx::={ }_{\|}^{\|}={ }_{\Pi}^{\|}={ }_{\Pi}^{\|}=,
$$

where again the contraction dimension $\chi$ is used to control the error of the approximation. With the CTMs and HLTTs, the single-site density matrix can be written as

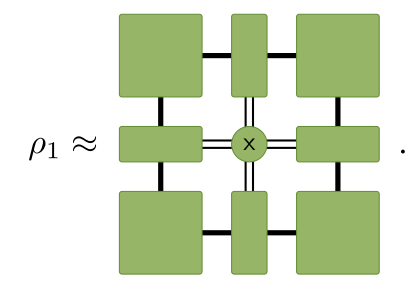

A powerful numerical tool to find CTMs and HLTTs is the so-called CTM renormalization group (CTMRG) $[23,24]$. The specific CTMRG procedure introduced in Ref. [31] is used in this work, as it is a particularly stable variant of CTMRG.

For this work both bMPS and CTMRG contractions have been implemented, and we observe that for equal $\chi$ both methods give almost identical results.

\section{B. Energy optimization}

As iPEPS are especially well suited to describe ground states, energy optimization algorithms for iPEPS are of particular interest. For almost a decade there was only a single strategy for this task: imaginary time evolution [22] in various variants. Recently, Refs. [32,33] introduced new direct variational approaches, which achieved lower energies than imaginary time evolutions, even in the limit of vanishing Trotter step size. Both variational methods rely on interaction contractions, similar to the norm contractions described in the previous section, but including all interaction terms of the Hamiltonian.

The first method [32] makes use of CTMRG interaction contractions to formulate a generalized eigenvalue problem for a given iPEPS, where the eigenvector corresponding to the lowest eigenvalue is used to propose the next iPEPS tensor in the minimization run.

The second method [33] computes the energy gradient of an iPEPS from a bMPS interaction contraction, such that any gradient minimization technique can be used, e.g., conjugate gradient methods or quasi-Newton methods such as the Broyden-Fletcher-Goldfarb-Shanno (BFGS) algorithm [34-37].

However, it should be noted that CTMRG interaction contractions can also be used to obtain energy gradients and, vice versa, bMPS interaction contractions to obtain the optimization generalized eigenvalue problem [38].

For iPEPS energy optimizations in this work, bMPS interaction contractions in combination with the BFGS algorithm were primarily used, as this method turned out to be the most stable one. Also, the BFGS algorithm seems to be more stable close to the minimum compared to conjugate gradient methods. Some states also have been optimized using a brute force minimization method, i.e., by computing finite difference energy gradients. All iPEPS optimized in this work have a single-site unit cell with a complex tensor which was forced to be invariant under spatial rotations. No symmetries at the virtual level were imposed. It turned out that starting with several random states and small contraction dimensions $(16 \lesssim \chi \lesssim 32)$ is the most economic way to bootstrap energy minimizations. The contraction dimension is then successively increased-in our optimizations up to values of $\chi=512$. For the transverse-field Ising model we observed that it can also be beneficial to use intermediate minimization results to initialize minimizations for nearby values of the transverse field.

\section{Correlation length}

A correlation function $c(r)$ of two arbitrary observables can be written as

$$
c(r)=u(1 /)^{r-1}=\boldsymbol{u}^{T} \boldsymbol{A}^{r-1} \boldsymbol{v},
$$

using a bMPS contraction, where the tensors $\boldsymbol{u}$ and $\boldsymbol{v}$ contain the specific observables. It should be noted that the same correlation function can be expressed by replacing the bMPS tensors with HLTTs from a CTMRG contraction.

We write the eigendecomposition of the transfer matrix as

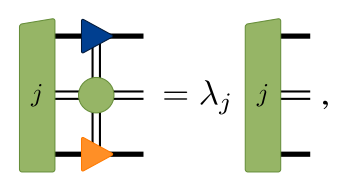

with $\left|\lambda_{0}\right| \geq\left|\lambda_{1}\right| \geq \ldots$ and without loss of generality we assume $\lambda_{0}=1$. Then the dominant correlation length of the system $\xi$ can be extracted as

$$
\xi=-\frac{1}{\log \left|\lambda_{1}\right|} .
$$

With this method the dominant correlation length can be extracted without even knowing which observables lead to the corresponding correlation function.

It should be noted that in contrast to local observables, the correlation length requires huge contraction dimensions $\chi$ to converge. Incorporating the ideas of Ref. [39], we observe the functional behavior, 


$$
\frac{1}{\xi(\chi)}=\frac{1}{\xi(\infty)}+k \log \left|\frac{\lambda_{1}(\chi)}{\lambda_{2}(\chi)}\right|
$$

which enables us to extract a converged value for the correlation length $\xi(\infty)$ precisely already for moderate values of $\chi$. In cases where $\lambda_{1} \simeq \lambda_{2}$ (due to degeneracy, as observed, e.g., in the $S=1 / 2$ Heisenberg model), one should use the largest eigenvalue different from $\lambda_{1}$ instead of $\lambda_{2}$ for the scaling in Eq. (15).

\section{QUANTUM CRITICAL BEHAVIOR IN A $(2+1) d$ CONFORMAL FIELD THEORY}

In a first application we study the critical behavior of our variationally optimized iPEPS tensors for a quantum critical point in the $(2+1) d$ Ising universality class. In the following we call this universality class described by a $(2+1) d$ dimensional, Lorentz-invariant conformal field theory the $3 d$ Ising CFT. Note that this critical behavior is distinct from the one observed in the so-called Ising PEPS [21], which amounts to promoting the thermal partition function of the 2D classical Ising model into a twodimensional quantum many-body wave function in PEPS form with bond dimension $D=2$. This wave function can be parametrized by the temperature $T$ entering the partition function, and at $T=T_{c}$ describes a critical wave function with algebraically decaying correlation functions. However, the critical properties of this wave function are described by the 2D Ising CFT.

\section{A. Overview}

The Hamiltonian studied in this section is the transversefield Ising model on an infinite square lattice with an additional longitudinal magnetic field,

$$
H_{\mathrm{TFI}}=-J \sum_{\langle i, j\rangle} \sigma_{i}^{z} \sigma_{j}^{z}-h \sum_{i} \sigma_{i}^{x}-h_{z} \sum_{i} \sigma_{i}^{z}
$$

where $J=1$ denotes the strength of the ferromagnetic Ising interactions and sets the energy scale, $h$ denotes the transverse field while $h_{z}$ parametrizes the longitudinal magnetic field. The phase diagram of this model is sketched in Fig. 1. For $h_{z}=0$ the model has a $\mathbb{Z}_{2}$ spin-inversion symmetry and features a quantum phase transition at $h / J=$ $h_{c}=3.04438(2)$ [40], which separates a paramagnetic phase with $m_{z} \equiv\left\langle\sigma_{i}^{z}\right\rangle=0$ for $h>h_{c}$ from a symmetry broken phase with $m_{z} \neq 0$ for $h<h_{c}$. The quantum critical point at $h=h_{c}, h_{z}=0$ is described by the 3D Ising CFT. For all finite $h_{z} \neq 0$ there is no critical behavior and the $z$ magnetization $m_{z}$ is finite as a response to the finite $h_{z}$. In the entire phase diagram with $h \neq 0$, the transverse $x$ magnetization $m_{x} \equiv\left\langle\sigma_{i}^{x}\right\rangle$ is finite.

In the following we explore the physics in the vicinity of the critical point $h=h_{c}, h_{z}=0$ using iPEPS simulations. In order to provide field theoretical predictions to compare

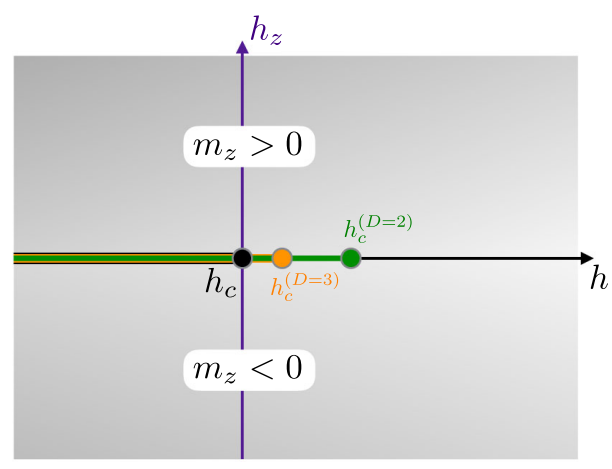

FIG. 1. Phase diagram of the ferromagnetic $(J=1)$ transversefield Ising model with an additional longitudinal field $h_{z}$. The thick, colored lines for $h<h_{c}$ or $h<h_{c}^{(D)}$ highlight the horizontal extent of the spontaneous symmetry-breaking line at $h_{z}=0$.

with, we briefly review the properties of the 3D Ising CFT for our purposes. The 3D Ising CFT has two relevant perturbations, the $O_{\sigma}$ and the $O_{\epsilon}$ field. Their scaling dimensions $\Delta_{\sigma}$ and $\Delta_{\epsilon}$ are given in Table I. They clearly differ in $2 d$ and $3 d$, which leads to distinct critical behavior. In the transverse-field Ising model it is expected that in the continuum limit we can identify $\sigma_{i}^{z} \sim O_{\sigma}\left(\boldsymbol{r}_{i}\right)$, while $\sigma_{i}^{x} \sim O_{\epsilon}\left(\boldsymbol{r}_{i}\right)+$ const.

We perturb a general CFT in $d$ space-time dimensions with a relevant perturbation $\phi$ with scaling dimension $\Delta_{\phi}$ :

$$
\mathcal{H}(\lambda)=\mathcal{H}_{\mathrm{CFT}}+\lambda \int d x^{d-1} \hat{\phi}
$$

Since the perturbation is relevant, i.e., $\Delta_{\phi}<d$, it opens a mass gap proportional to $\lambda^{1 /\left(d-\Delta_{\phi}\right)}$; respectively, it leads to a finite correlation length $\xi \sim \lambda^{-1 /\left(d-\Delta_{\phi}\right)} \equiv \lambda^{-\nu_{\phi}}$. In the transverse-field Ising model it is understood that the microscopic coupling $\left(h-h_{c}\right)$ couples to the field $O_{\epsilon}$, while $h_{z}$ couples to the field $O_{\sigma}$.

The 3D Ising CFT has two relevant perturbations, which translate into the two correlation length exponents,

TABLE I. Upper two rows: Relevant scaling dimensions of the Ising CFT in $d=2,3,4$ space-time dimensions; results for $d=3$ are from the most recent conformal bootstrap study [41]. The lower six rows denote the additional critical exponents probed in this work, which are derived from the scaling dimensions above.

\begin{tabular}{cclc}
\hline \hline Exponent & $d=2$ & \multicolumn{1}{c}{$d=3$} & $d=4$ \\
\hline$\Delta_{\sigma}$ & $1 / 8$ & $0.5181489(10)$ & 1 \\
$\Delta_{\epsilon}$ & 1 & $1.412625(10)$ & 2 \\
$\nu_{\sigma}=1 /\left(d-\Delta_{\sigma}\right)$ & $8 / 15$ & $0.4029250(2)$ & $1 / 3$ \\
$\nu_{\epsilon}=1 /\left(d-\Delta_{\epsilon}\right)$ & 1 & $0.629970(4)$ & $1 / 2$ \\
$\alpha_{\sigma}=\Delta_{\epsilon} \times \nu_{\sigma}$ & $8 / 15$ & $0.569182(4)$ & $2 / 3$ \\
$\alpha_{\epsilon}=\Delta_{\epsilon} \times \nu_{\epsilon}$ & 1 & $0.88991(1)$ & 1 \\
$\beta_{\sigma}=\Delta_{\sigma} \times \nu_{\sigma}$ & $1 / 15$ & $0.2087751(5)$ & $1 / 3$ \\
$\beta_{\epsilon}=\Delta_{\sigma} \times \nu_{\epsilon}$ & $1 / 8$ & $0.326418(2)$ & $1 / 2$ \\
\hline \hline
\end{tabular}


$\nu_{\epsilon}=1 /\left(d-\Delta_{\epsilon}\right)$ and $\nu_{\sigma}=1 /\left(d-\Delta_{\sigma}\right)$, which appear in the scaling relations $\xi \sim\left|h-h_{c}\right|^{-\nu_{\epsilon}}$ and $\xi \sim\left|h_{z}\right|^{-\nu_{\sigma}}$.

We are in a situation where the perturbed theory can display magnetizations, and we thus define critical exponents $\alpha_{\phi}, \beta_{\phi}$ which describe how the magnetizations grow as a function of the perturbing coupling for a perturbation $\phi . \alpha_{\phi}$ is the exponent we use when measuring $m_{x, c}-\left\langle\sigma_{i}^{x}\right\rangle \sim\left\langle O_{\epsilon}\right\rangle$, while we use $\beta_{\phi}$ for the $\left\langle\sigma_{i}^{z}\right\rangle \sim\left\langle O_{\sigma}\right\rangle$ observable. The subscript $\sigma, \epsilon$ denotes the perturbing field as for the correlation length exponents before. For the transverse magnetization we expect $\left|m_{x, c}-m_{x}\right| \sim|\lambda|^{\alpha_{\phi}}$, while for the longitudinal magnetization we expect $m_{z} \sim|\lambda|^{\beta_{\phi}}$. The definitions and values for these exponents can be found in Table I. Some of the exponents we introduce here for clarity reasons are also more commonly known as $\beta \equiv \beta_{\epsilon}, \delta \equiv 1 / \beta_{\sigma}$, and $\nu \equiv \nu_{\epsilon}$ in the statistical mechanics literature. Note that the $d=4$ critical exponents are equivalent to the mean-field exponents. This is because $d=4$ is the upper critical dimension for the Ising criticality, i.e., the dimension where mean-field theory becomes exact.
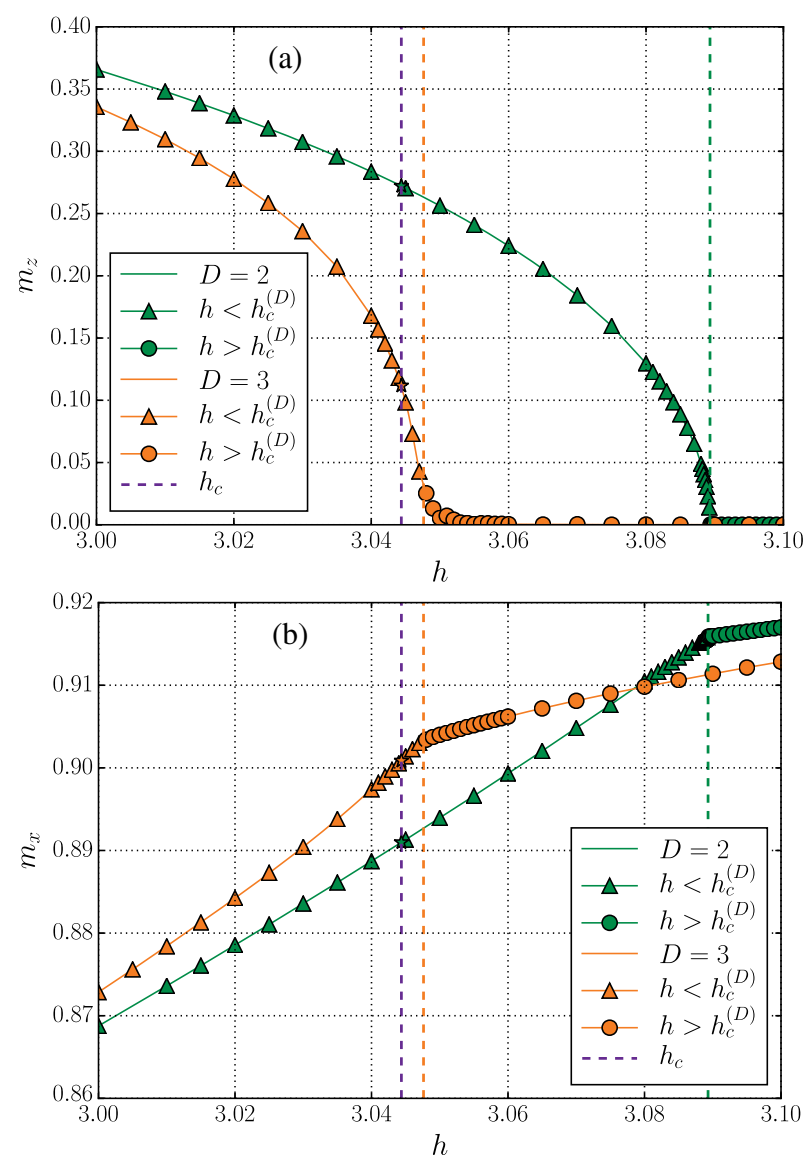

\section{B. iPEPS results for the transverse-field Ising model}

The transverse-field Ising model (with $h_{z}=0$ ) has been studied extensively with both finite-size PEPS and iPEPS approaches in the past [22,23,33,42-47]. Variational tensor network approaches have also been used to study the classical 3D Ising model, which is part of the same universality class $[48,49]$. A common feature of all these simulations is that for $h$ below a bond dimension $D$ dependent value $h_{c}^{(D)}$, the system shows a finite $z$ magnetization $m_{z}$. However, in the past the values of $h_{c}^{(D)}$ and the functional behavior of $m_{z}$ in its vicinity did depend significantly on the tensor optimization methodology. We believe the newest generation of optimization algorithms put forward in Refs. [32,33] does not suffer from these shortcomings anymore, so that a detailed analysis of the intrinsic iPEPS finite- $D$ behavior close to the 3D Ising CFT is finally possible.

\section{Magnetizations in the vicinity of the critical point}

We start by presenting in Fig. 2 the behavior of the $z$ and the $x$ magnetization at $h_{z}=0$ by varying $h$ in Figs. 2(a)
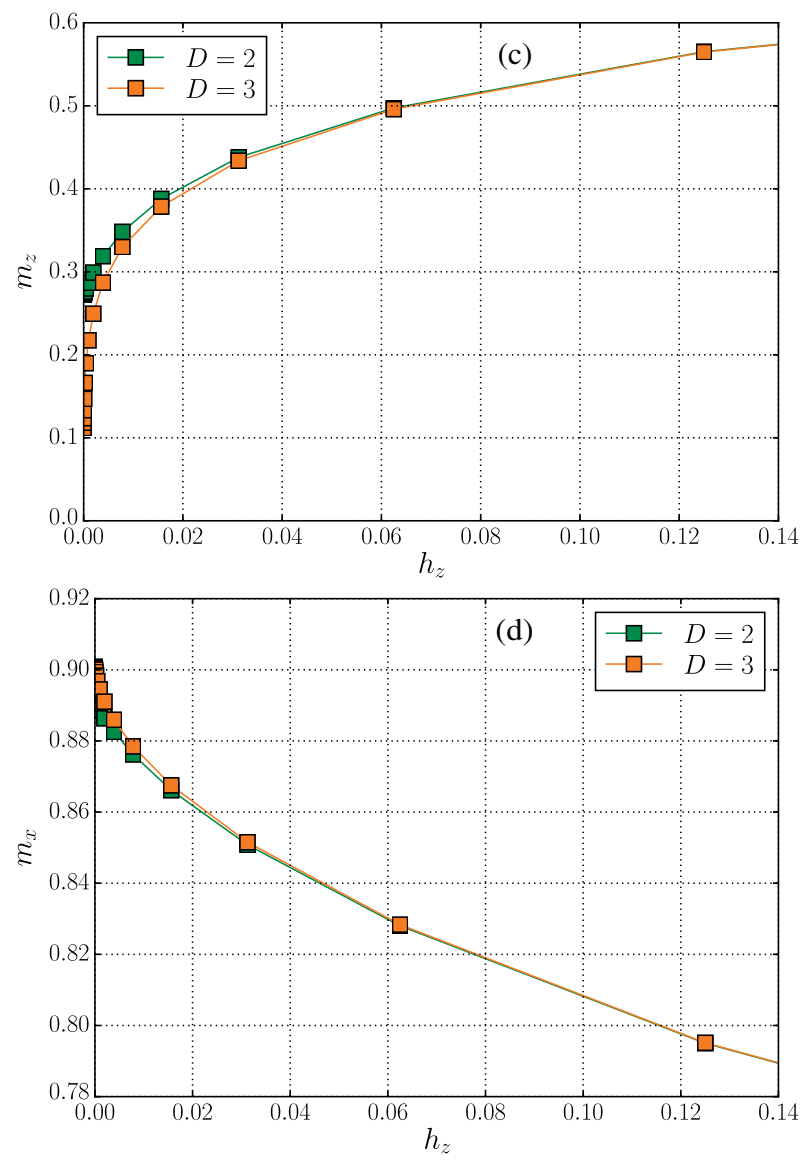

FIG. 2. iPEPS results for the magnetizations per site $m_{z}=\left|\left\langle\sigma_{i}^{z}\right\rangle\right|$ and $m_{x}=\left\langle\sigma_{i}^{x}\right\rangle$ as a function of $h\left(h_{z}=0\right)$ in (a) and (b) and as a function of $h_{z}\left(h=h_{c}\right)$ in (c) and (d). Result for iPEPS bond dimension $D=2,3$ are shown. The vertical dashed lines indicate the critical values of $h_{c}^{(D)}$ for both $D$ values, as well as the literature reference for $h_{c}$ [40]. 
and 2(b) for bond dimensions $D=2,3$. As previously reported, we also observe a $D$-dependent value $h_{c}^{(D)}$, where $m_{z}$ vanishes, while $m_{x}$ displays a kink. We find $h_{c}^{(D=2)} \approx 3.0893$, while $h_{c}^{(D=3)} \approx 3.0476$. Note that the $D=3$ result differs only by about one part per thousand from the reference critical value, $h_{c}=3.04438(2)$ [40]. In an earlier study based on one-dimensional iMPS states for the $(1+1) d$ transverse-field Ising model, a bond dimension $D>10$ was required to reach a comparable accuracy [44]. We have also tried to optimize $D=4$ tensors, but albeit technically possible, it turns out to be extremely difficult to obtain energies which are lower than our best $D=3$ results so far. We come back to this observation later in this section. Finally, in Figs. 2(c) and 2(d), we display $m_{z}$ and $m_{x}$ along an orthogonal cut at fixed $h=h_{c}$ with varying $h_{z}>0$, i.e., along the violet axis in Fig. 1.

While the plots in Fig. 2 seem to suggest large differences between $D=2$ and $D=3$, it should be noted that the $h$ and $h_{z}$ ranges shown are already quite small. Shown on a scale $h \in[0,4]$ it would be difficult to visually spot the differences between the two $D$ values.
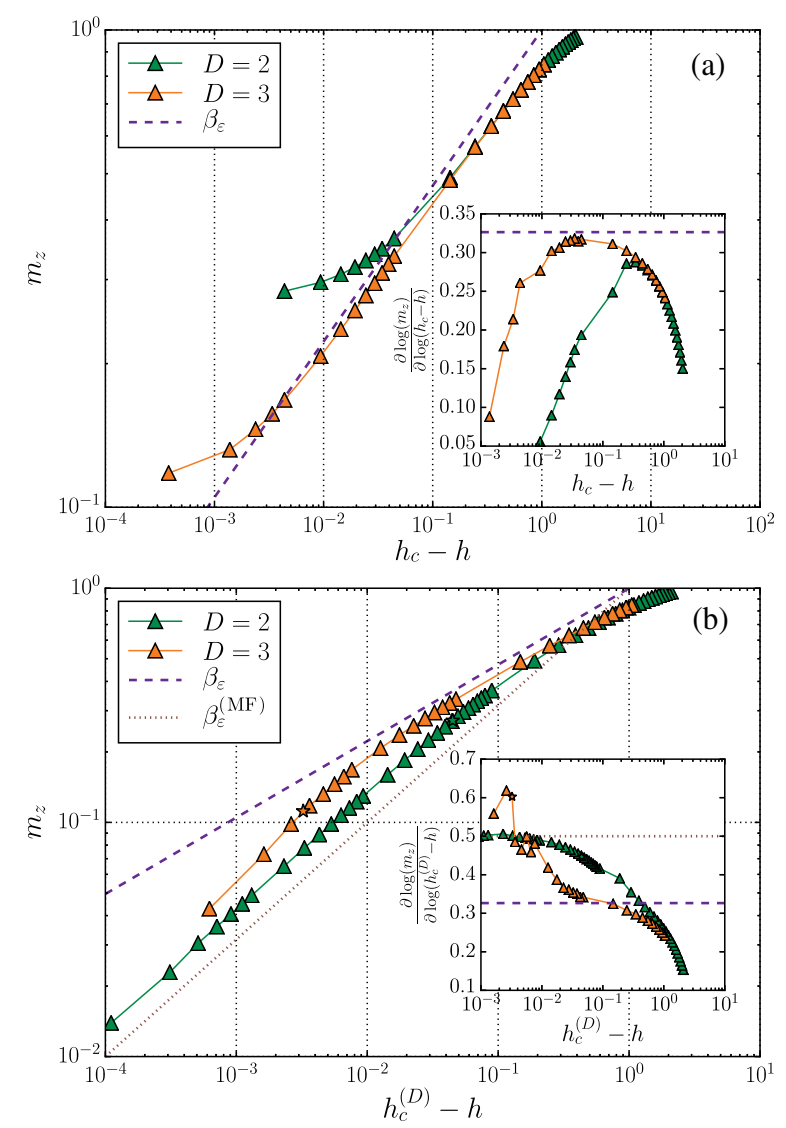

\section{Critical exponents}

In a next step we explore the critical behavior contained in the presented data. In Fig. 3(a), we plot $m_{z}$ as a function of $h_{c}-h$ on a log-log scale. For comparison we plot a straight line with the expected slope $\beta_{\epsilon}$ as a guide to the eye. In the corresponding inset we numerically calculate the derivative and find a collapse between the $D=2$ and $D=3$ data at larger distances from the critical point. The $D=2$ running estimate for the critical exponent reaches a maximum of $\sim 0.29$ and then drops to zero as $h_{c}-h$ goes to zero. The $D=3$ running estimate rises to $\sim 0.32$ before it also drops to zero as $h_{c}-h$ goes to zero. We expect that $D>3$ would get even closer to the expected $\beta_{\epsilon} \approx 0.326418(2)$, before dropping to zero as $h_{c}-h$ goes to zero. The drop to zero is clearly due to the finite- $D$ remnant $m_{z}$ at the thermodynamic value $h_{c}$. Let us therefore investigate what happens when we analyze the data as a function of $h_{c}^{(D)}-h$ instead. Figure 3(b) displays the corresponding data. The $D=2$ data clearly show a limiting mean-field behavior $\beta_{\epsilon}^{(\mathrm{MF})}=1 / 2$ at small $h_{c}^{(D)}-h$ (in the inset), as observed previously in Ref. [44]. The $D=3$ data
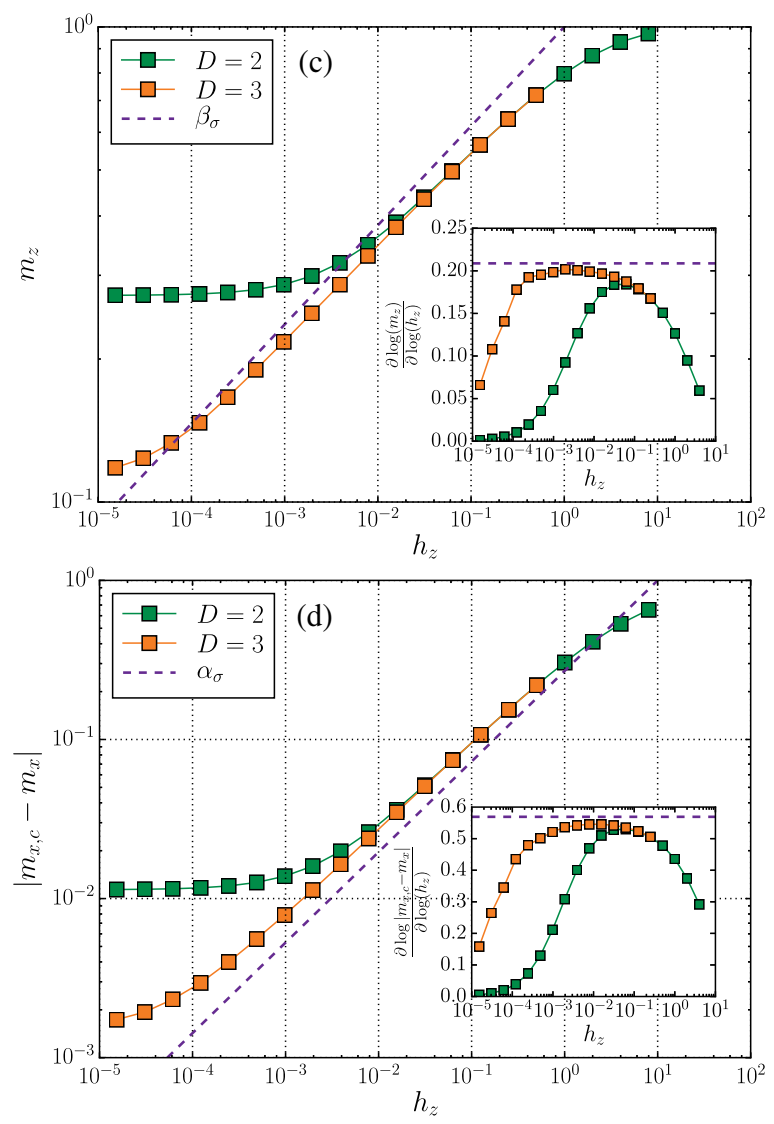

FIG. 3. Analysis of the critical behavior of the $m_{z}$ and $m_{x}$ magnetizations in the vicinity of the critical point at $h=h_{c}, h_{z}=0$. (a) $m_{z}$ as a function of $h_{c}-h$; inset shows good convergence to the expected $\beta_{\epsilon}$ exponent. (b) $m_{z}$ as a function of $h_{c}^{(D)}-h$; inset shows crossover to mean-field behavior. (c) $m_{z}$ as a function of $h_{z}$; inset shows good convergence to the expected $\beta_{\sigma}$ exponent. (d) $\left|m_{x}-m_{x, c}\right|$ as a function of $h_{z}$; inset shows good convergence to the expected $\alpha_{\sigma}$ exponent. The expected exponents are shown in Table I. 
show some hint of an intermediate plateau around the true 3D Ising CFT value for $\beta_{\epsilon}$ before also crossing over to the mean-field value $\beta_{\epsilon}^{(\mathrm{MF})}$ at small $h_{c}^{(D)}-h$. The analysis of the $m_{x}$ magnetization is less clean and shown in Fig. 11 in Appendix A. In Figs. 3(c) and 3(d), we present the analogous analysis for both $m_{z}$ and $m_{x}$ when staying at $h=h_{c}$ while tuning $h_{z}>0$. The numerical derivatives provide running averages for $\beta_{\sigma}$ and $\alpha_{\sigma}$ which converge nicely towards the expected values as we increase $D$ from 2 to 3 . The maximal values for $D=3$ are only a few percent below the 3D Ising CFT results.

So we learn that when the location of the critical point is known beforehand, the critical behavior can be determined quite accurately already with a surprisingly small bond dimension of $D=3$. In the vicinity of the finite- $D$ critical points we, however, observe mean-field behavior, and due to the crossover it is more difficult to extract the genuine critical behavior.

\section{Correlation lengths}

After having analyzed the critical behavior of local observables as a function of perturbing couplings, we now investigate the correlation lengths in our optimized iPEPS wave functions in the vicinity of the critical point. In the vicinity and at a quantum critical point in $(1+1) d$ represented with a finite bond dimension iMPS, we know that only a finite correlation length can appear. Since iPEPS is in principle able to represent wave functions with algebraically decaying correlations, i.e., states with infinite correlation lengths [21], it is not a priori clear what to expect in our optimized iPEPS wave functions. Let us note first that the correlation lengths for $D=1$ (product states) vanish identically, even though the spontaneous magnetization shows critical mean-field behavior at $h_{c}^{(D=1)}=4$ (not shown). Based on the technology presented in Sec. II C, we have determined the largest correlation lengths for $D=2$ and $D=3$ iPEPS states along the previously investigated cuts in the $\left(h, h_{z}\right)$ plane. The results are shown in Figs. 4(a) and 4(c). The observed correlation lengths for $D=2$ do not grow beyond $\xi_{\max }^{(D=2)} \approx 1.67$ lattice spacings, and they reach their maximum at $h=h_{c}^{(D=2)}, h_{z}=0$. We are quite confident that this short correlation length is not an artifact of incomplete optimization, but is a genuine feature of optimized, translationally invariant, finite- $D$ iPEPS wave functions for Lorentz-invariant quantum critical systems with a 3D space-time description. For $D=3$ we also observe finite correlation lengths, but now the maximum is substantially larger: $\xi_{\max }^{(D=3)} \approx 12.2$ at $h=h_{c}^{(D=3)}, h_{z}=0$ [50]. So both $D$ values seem to indicate that our variational optima feature a finite correlation length. This is one of the key results of this paper, whose possible origin we are going to discuss later. We do, however, show in the following that
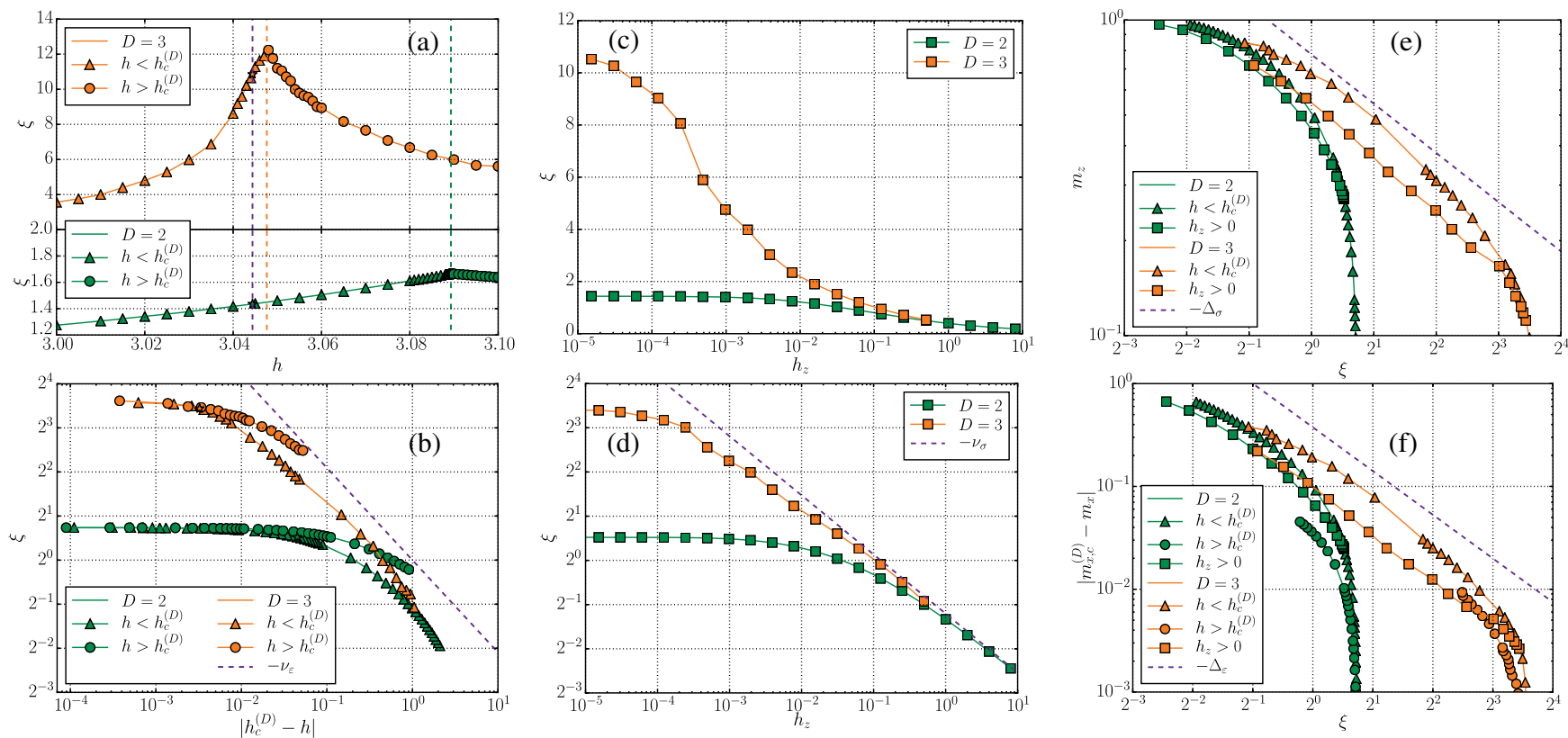

FIG. 4. Correlation lengths of the variationally optimized iPEPS states for $D=2$ and $D=3$. (a) Correlation lengths as a function of the transverse field $h$. (b) Correlation lengths as function of $\left|h_{c}^{(D)}-h\right|$ on a log-log scale, including the theoretically expected slope $-\nu_{\epsilon}$. (c) Correlation lengths as a function of the longitudinal field $\left|h_{z}\right|$. (d) Correlation lengths as function of $\left|h_{z}\right|$ on a log-log scale, including the theoretically expected slope $-\nu_{\sigma}$. Panels (e) and (f) display the expectation value of $m_{z}$ and $\left|m_{x, c}^{(D)}-m_{x}\right|$, respectively, as a function of the correlation length $\xi$, on a logarithmic scale. The expected slopes in the two cases are directly the (negated) scaling dimensions $-\Delta_{\sigma}$ and $-\Delta_{\epsilon}$. The values of the exponents and scaling dimensions can be found in Table I. 
the finite correlation length is also a benefit, as it helps us to understand and organize the finite- $D$ effects in field theoretically motivated formulas based on $\xi(D)$.

Before doing this, let us investigate the functional behavior of the correlation length in the vicinity of the critical point. Depending on the cut in parameter space, we expect $\xi \sim\left|h_{c}-h\right|^{-\nu_{\epsilon}}$ or $\xi \sim\left|h_{z}\right|^{-\nu_{\sigma}}$, with the values of $\nu_{\epsilon}$ and $\nu_{\sigma}$ given in Table I. Indeed, the data in Figs. 4(b) and 4(d) shown on a log-log scale are (roughly) compatible with the expected correlation length exponents in some intermediate window of the couplings. This is expected since far away from the critical point we are outside the quantum critical regime, while very close to the critical point $\xi$ saturates. Still, the agreement for the $h_{z}$ detuning is much better than the $\left|h_{c}-h\right|$ detuning.

Finally we plot the expectation values of the two magnetizations $m_{z}$ and $\left|m_{x, c}-m_{x}\right|$ as a function of the measured correlation length $\xi$ (for both parameter cuts) in Figs. 4(e) and 4(f). As discussed earlier, we expect this relation to be governed by the scaling dimensions $\Delta_{\sigma}$ and $\Delta_{\epsilon}$, respectively. While the $D=2$ results do not match too well, the $D=3$ results for both parameter cuts and both observables are in good agreement with the expected scaling dimensions.

Even though it seems that the critical exponents and scaling dimensions can be obtained more precisely based on the observables as a function of the couplings than of the correlation lengths, it is nevertheless rewarding to observe that the correlation lengths are also following the expected quantum critical behavior with increasing $D$, within the stated limitations.

\section{Critical energy convergence}

We now investigate the energy convergence of the TFI model at its critical point $h=h_{c}, h_{z}=0$ for increasing bond dimension $D$. It is one of the open problems in practical iPEPS calculations to understand the convergence of energies as a function of $D$. Here we advocate that the variational energy of an optimized iPEPS tensor at the critical point $h=h_{c}, h_{z}=0$ can be understood as a particular type of a Casimir effect controlled by the correlation length $\xi$.

It is well known that the ground-state energy density $e=E / N_{\text {sites }}$ of a $3 \mathrm{D}$ quantum critical system in a torus geometry with modular parameter $\tau$ [51] is given as

$$
e(L)=e(\infty)-\frac{\alpha_{\mathrm{QCP}}^{(\tau)} \times v}{L^{3}}+\cdots,
$$

where $L$ denotes the linear extent of the torus, $v$ is the "speed of light," e.g., the critical spin wave velocity in a TFI model, and $\alpha_{\mathrm{QCP}}^{(\boldsymbol{\tau})}$ is a $\boldsymbol{\tau}$-dependent Casimir amplitude which otherwise depends only on the universality class of the quantum critical point (QCP); for example, for the 3D Ising CFT and a square torus $(\boldsymbol{\tau}=\boldsymbol{i})$ [52] with periodic boundary conditions, $\alpha_{3 \mathrm{D} \text { Ising CFT }}^{(\tau=i)}=+0.35(2)$, according to Ref. [53].
We now postulate that our iPEPS setup can be considered as a new, distinct geometry with its own Casimir amplitude, $\alpha_{3 \mathrm{D} \text { Ising CFT }}^{\text {(iPEPS }}$, where, however, the length of the torus is replaced by the correlation length $\xi$, such that

$$
e(\xi)=e(\infty)-\frac{\alpha_{3 \mathrm{D} \mathrm{Ising} \mathrm{CFT}}^{(\mathrm{iPES})} \times v}{\xi^{3}}+\cdots
$$

We stress that this ansatz is in agreement with the expected scaling behavior of the one-point function of the stressenergy tensor $T$, whose scaling dimension in $d=3$ is $\Delta_{T}=3$. In the past a similar scaling with a $1 / \xi^{2}$ dependence has been suggested and numerically verified in the $(1+1) d$ iMPS context in Ref. [8]. Since we only have two values of $D$, it is a priori hard to determine the validity of the postulated energy convergence form. Let us nevertheless use the best variational energies at $h=h_{c}, h_{z}=0$ for $D=2$ and $D=3$, together with the literature value of $v=3.323(33)[51]$ to estimate

$\alpha_{3 \mathrm{D} \text { Ising CFT }}^{(\mathrm{iPEP})} \approx-0.00061, \quad e_{\mathrm{TFI}}^{(\mathrm{iPEPS}, \infty)} \approx-3.2342623$.

If correct, the scaling hypothesis Eq. (18) combined with the very small iPEPS Casimir amplitude would explain the spectacular accuracy of the $D=3$ results. The $D=3$ correlation length of beyond 10 lattice sites gives an energy correction proportional to $1 / \xi^{3} \lesssim 10^{-3}$, while the Casimir amplitude $\alpha_{3 \mathrm{D} \text { Ising CFT }}^{\text {(iPEP) }}$ is itself 3 orders of magnitude smaller than the square torus amplitude. So multiplying these two factors we are led to conjecture that the extrapolated iPEPS energies are accurate to about $10^{-6}$

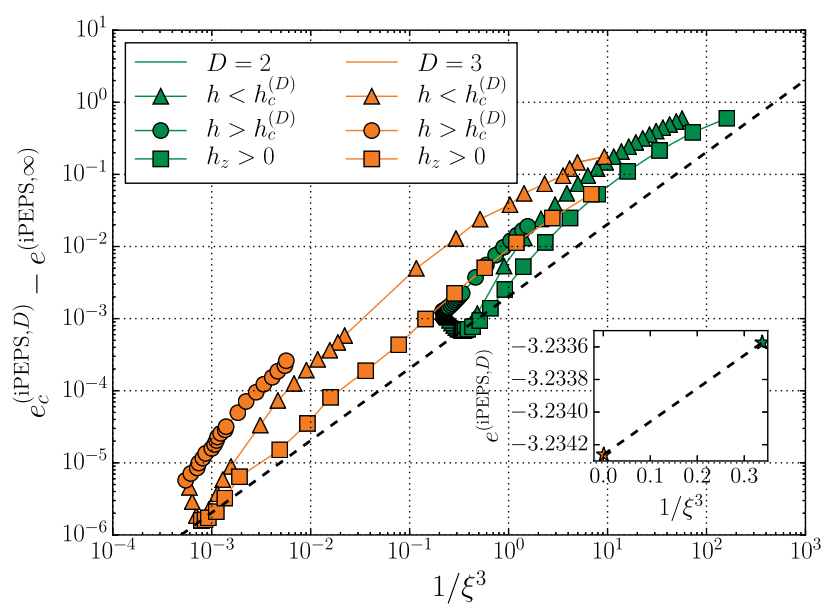

FIG. 5. Variational excess critical energy density $\Delta e_{c}=$ $e_{c}^{(\mathrm{iPEPS}, D)}-e_{c}^{(\mathrm{iPEPS}, \infty)}$ of transverse-field Ising iPEPS wave functions optimized for various $\left(h, h_{z}\right)$ as a function of their $1 / \xi^{3}$. The variational excess energy density corroborates the advocated $1 / \xi^{3}$ scaling. Inset: Fit according to Eq. (18), yielding $e_{c}^{(\mathrm{iPEPS}, \infty)}$ and $\alpha_{3 \mathrm{D} \text { Ising CFT }}^{\text {(iPEPS }}$ in Eq. (19). 
already at $D=3$. This result might explain why $D=4$ simulations are so demanding, as the expected remaining energy gains are tiny and are accompanied with wave functions bestowed with large correlation lengths, which are even harder to contract accurately.

In order to corroborate the advocated scaling ansatz in Eq. (18), we plot the critical (i.e., evaluated at $h=h_{c}$, $h_{z}=0$ ) variational energies $e_{c}^{(\mathrm{iPEPS}, D)}$ of all available iPEPS wave functions in a common plot, cf. Fig. 5. In the inset we show the fit according to Eq. (18) of the two data points with the best $D=2$ and $D=3$ critical energies. The data in the main plot are seen to approximately scale according to $\Delta e_{c} \propto 1 / \xi^{3}$ over several orders of magnitude, thus providing nontrivial empirical evidence in favor of the validity of the ansatz Eq. (18) [54].

\section{CONTINUOUS SYMMETRY BREAKING}

In this section, we want to explore the properties of iPEPS wave functions as they represent or approximate quantum many-body states which exhibit continuous symmetry breaking in $(2+1)$ space-time dimensions. This is a rather ubiquitous phenomenon, ranging from magnetic order in $O(N)$ symmetric quantum magnets with $N \geq 2$, to bosonic and fermionic superfluids, to superconductors and Goldstone phases in high-energy physics. In these systems the continuous symmetry is spontaneously broken, accompanied by the appearance of a finite-order parameter $|\mathbf{M}|>0$. Another hallmark is the required presence of gapless excitations (so-called Goldstone modes), which are the soft long-wavelength modes of order parameter variations. We study the $S=1 / 2$ Heisenberg antiferromagnet and the $S=1 / 2 X Y$ model, both on the square lattice, as paradigmatic examples for $O(3)$ and $O(2)$ continuous symmetry breaking with a three- and two-component, respectively, vector order parameter.

\section{A. Overview}

The field theoretical description of collinear magnetic order in $O(N)$ quantum magnets relies often on a quantum nonlinear sigma model (NLSM) formulation, or on a description in terms of the symmetry-breaking phase in an $N$-component interacting $\phi^{4}$ theory. The gapless Goldstone modes are also known as spin waves in the magnetic context, and taken in isolation they behave as a collection of free massless scalar fields. These gapless modes are responsible, e.g., for the algebraic decay of spin-spin correlations to their limiting value and the finite-size corrections of the energy or the order parameter. These linearly dispersing modes require a $(2+1) \mathrm{D}$ Lorentz-invariant description at low energies, similar to the Ising CFT discussed before.

The quantum nonlinear $O(N)$ sigma model is described in detail in Ref. [55]. For our purpose it is sufficient to know that this is basically a hydrodynamic theory of quantum magnets with collinear order. Its description, being hydrodynamic, relies only on a handful of effective parameters entering the description: the spin stiffness $\rho_{s}$, the spin wave velocity $v$, the transverse susceptibility $\chi_{\perp}$, and the squared order parameter in the thermodynamic limit $m^{2}(\infty)$. The first three parameters are actually related via $v^{2}=\rho_{s} / \chi_{\perp}$. Similar to the finite-size corrections to the ground-state energy discussed in the quantum critical context, the finite-size corrections to the ground-state energy and the order parameter have been derived for the quantum nonlinear $O(N)$ sigma model in Refs. [5660]. The finite-size corrections to the ground-state energy $e=E / N_{\text {sites }}$ in $d=3$ are as follows:

$$
\begin{aligned}
e(L)= & e(\infty)-\left[\alpha_{\mathrm{NLSM}}^{\text {(shape } / \mathrm{bc})}\left(\frac{N-1}{2}\right) v\right] \frac{1}{L^{3}} \\
& +\frac{(N-1)(N-2)}{8} \frac{v^{2}}{\rho_{s} L^{4}}+\mathcal{O}\left(\frac{1}{L^{5}}\right) .
\end{aligned}
$$

For a square torus with periodic boundary conditions, $\alpha_{\text {NLSM }}^{(\tau=i)} \approx 1.437745$ has been obtained. The finite-size correction for the magnetic order parameter squared are as follows:

$\frac{m^{2}(L)}{m^{2}(\infty)}=1+\left[\mu_{\mathrm{NLSM}}^{\text {(shape } / \mathrm{bc})}\left(\frac{N-1}{2}\right) \frac{v}{\rho_{s}}\right] \frac{1}{L}+\mathcal{O}\left(\frac{1}{L^{2}}\right)$.

For a square torus with periodic boundary conditions, $\mu_{\mathrm{NLSLM}}^{(\tau=i)} \approx 0.62075$ has been found.

As we see below, our variationally optimized iPEPS wave functions have a finite correlation length $\xi$, which depends on the model and on the bond dimension $D$. We now conjecture the following $d=3$, finite $\xi$ corrections for the ground-state energy density $e$ and the magnetic order parameter squared $\mathrm{m}^{2}$ :

$e(\xi)=e(\infty)-\left[\alpha_{\mathrm{NLSM}}^{(\mathrm{iPES})}\left(\frac{N-1}{2}\right) v\right] \frac{1}{\xi^{3}}+\mathcal{O}\left(\frac{1}{\xi^{4}}\right)$

and

$\frac{m^{2}(\xi)}{m^{2}(\infty)}=1+\left[\mu_{\mathrm{NLSM}}^{(\mathrm{iPEPS})}\left(\frac{N-1}{2}\right) \frac{v}{\rho_{s}}\right] \frac{1}{\xi}+\mathcal{O}\left(\frac{1}{\xi^{2}}\right)$.

The potential power of these formulas lies in the fact that one can extrapolate the results at finite $\xi(D)$ to the limit $\xi \rightarrow \infty$ based on iPEPS data fits to the above formulas. Furthermore, it is possible to predict the finite-size corrections for other microscopic models, once the "universal" values of $\alpha_{\mathrm{NLSM}}^{(\mathrm{iPES})}$ and $\mu_{\mathrm{NLSM}}^{(\mathrm{iPEPS})}$ are determined. The knowledge of $N, v$, and $v / \rho_{s}$ allows us then to quantitatively predict the slope of the finite-size corrections. Conversely, it might become possible to estimate $v$ and $v / \rho_{s}$ based on precise iPEPS data for a model with a known value for $N$. 


\section{B. $S=1 / 2$ antiferromagnetic Heisenberg model}

The $S=1 / 2$ antiferromagnetic Heisenberg model has also been studied frequently using finite-size PEPS and iPEPS approaches in the past $[32,33,42,45,61-63]$. The ground state of this model has antiferromagnetic Néel order, which breaks the continuous $O(N=3)$ rotation symmetry spontaneously down to a residual $O(2)$ symmetry. The presence of $N-1=2$ Goldstone modes leads to an algebraic decay of the two-spin correlation function.

The Hamiltonian is defined as

$$
H_{\mathrm{HB}}=J \sum_{\langle i, j\rangle}\left(S_{i}^{x} S_{j}^{x}+S_{i}^{y} S_{j}^{y}+S_{i}^{z} S_{j}^{z}\right)
$$

with $J=1$ and the $S_{i}^{\alpha}$ are spin- $1 / 2$ operators. In order to be able to work with a single-site unit cell, we perform a spin rotation on one Néel sublattice, which negates the sign of the $y$ and $z$ parts of the interactions in the actual calculations.

We proceed optimizing the variational energies of iPEPS tensors for $D=2,3,4,5$. Then we measure the order parameter squared $m^{2}=\left|\left\langle\boldsymbol{\sigma}_{i}\right\rangle\right|^{2}$ (i.e., the maximum possible for the order parameter squared amounts to one), as well as the correlation length $\xi$. These correlation lengths are finite and range from $\xi(D=2) \approx 0.7$ to $\xi(D=5) \approx 4.5$. The correlation lengths are thus substantially smaller than those of the critical transverse-field Ising model at $D=3$.

In Fig. 6, we present the energy per site $e$ as a function of $1 / \xi^{3}$ in the left-hand panel and $m^{2}$ as a function of $1 / \xi$ in the right-hand panel. This is the conjectured $\xi$ scaling form of Eqs. (22) and (23). It is striking that for both observables a linear fit leads to accurate extrapolations to the limit $\xi \rightarrow \infty$, when compared to high-precision quantum
Monte Carlo (QMC) reference values [56,64], $e_{\mathrm{HB}}^{(\mathrm{QMC})}=$ $-0.669437(5)$ and $m_{\mathrm{HB}}^{2(\mathrm{QMC})}=0.3770(8)$. We fit the largest three $D$ values for the energy $e$ and all the $D$ values for $m^{2}$ and obtain the following iPEPS $\xi \rightarrow \infty$ estimates: $e_{\mathrm{HB}}^{(\mathrm{PPES})}=$ $-0.66942(2)$ and $m_{\mathrm{HB}}^{2(\mathrm{iPEPS})}=0.380(4)$. Using the iPEPS fit slopes, the value $N=3$, and the known QMC values of the hydrodynamic parameters $v$ and $v / \rho_{s}[56,65]$, we can then proceed to determine

$$
\alpha_{\mathrm{NLSM}}^{(\mathrm{iPEPS})} \approx-0.0029
$$

and

$$
\mu_{\mathrm{NLSM}}^{(\mathrm{iPEPS})} \approx+0.045 .
$$

Note that similar to the Ising Casimir amplitude, the NLSM energy Casimir amplitude is 3 orders of magnitude smaller than the square torus one, highlighting that an iPEPS calculation at a certain $\xi$ should be considered 3 orders of magnitude more accurate than a square torus with $L \sim \xi$. The iPEPS order parameter amplitude is, however, only 1 order of magnitude smaller than the square torus result.

\section{C. $S=1 / 2 X Y$ model}

The $S=1 / 2 X Y$ model has also been investigated with PEPS and iPEPS in the past $[33,61,66]$. The ground state of this model has ferromagnetic order in the $x-y$ spin plane, which breaks the continuous $O(N=2)$ in-plane rotation symmetry spontaneously down to a residual discrete $\mathbb{Z}_{2}$ symmetry. The presence of $N-1=1$ Goldstone mode leads to an algebraic decay of the two-spin correlation function.
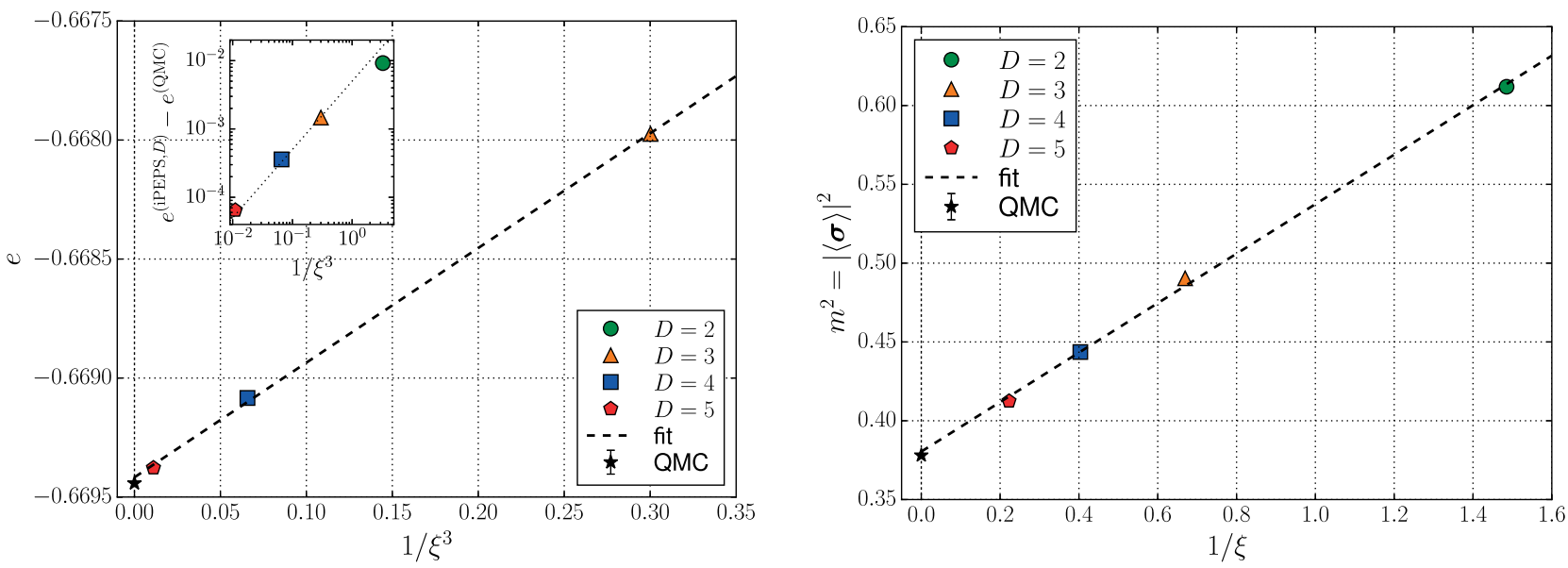

FIG. 6. $S=1 / 2$ Heisenberg antiferromagnet: iPEPS data for the ground-state energy per site $e$ (left-hand panel) and the order parameter squared $m^{2}$ (right-hand panel). We plot the data as a function of the expected $1 / \xi^{3}$ (for $e$ ) and $1 / \xi$ (for $m^{2}$ ) dependence. The linear fits to the $D=3,4,5$ results in the left-hand and all $D \geq 2$ in the right-hand panel extrapolate closely to the high-precision quantum Monte Carlo reference results [56]. The inset in the left-hand panel highlights the overall $1 / \xi^{3}$ convergence of the energy per site. 

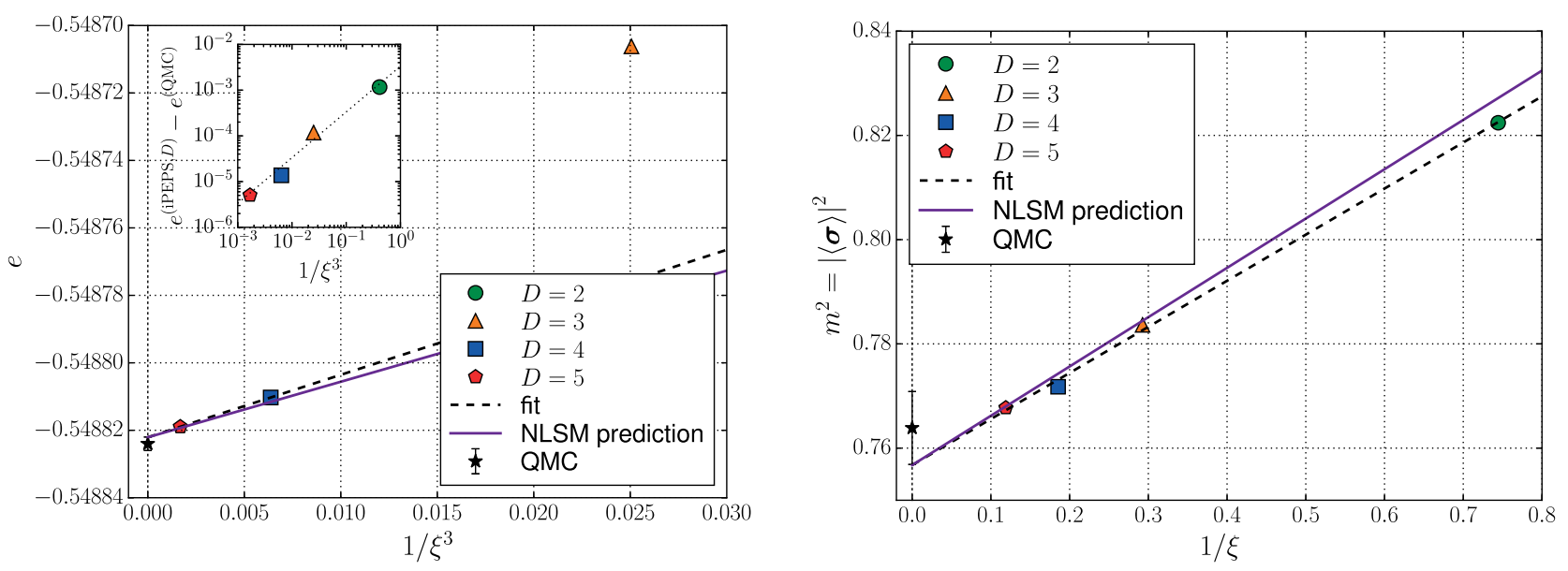

FIG. 7. $S=1 / 2 X Y$ ferromagnet: iPEPS data for the ground-state energy per site $e$ (left-hand panel) and the order parameter squared $m^{2}$ (right-hand panel). We plot the data as a function of the expected $1 / \xi^{3}$ (for $e$ ) and $1 / \xi$ (for $m^{2}$ ) dependence. The linear fits to the $D=4,5$ results in the left-hand and all $D \geq 2$ in the right-hand panel extrapolate reasonably closely to the quantum Monte Carlo results [68], and may well be more accurate than the somewhat antiquated QMC results. The inset in the left-hand panel highlights the overall $1 / \xi^{3}$ convergence of the energy per site. In both panels we include a prediction based on the conjectured finite $\xi$ formulas for the nonlinear sigma model (NLSM), cf. Eqs. (22) and (23) and the main text.

The Hamiltonian is defined as

$$
H_{X Y}=-J \sum_{\langle i, j\rangle}\left(S_{i}^{x} S_{j}^{x}+S_{i}^{y} S_{j}^{y}\right),
$$

with $J=1$. Note that for this model the two choices of the sign of $J$ can be mapped into each other. Since we want to use a single-site iPEPS unit cell, we adopt the ferromagnetic sign convention.

We proceed optimizing the variational energies of iPEPS tensors for $D=2,3,4,5$. Then we measure the order parameter squared, $m^{2}=\left|\left\langle\boldsymbol{\sigma}_{i}\right\rangle\right|^{2}$ (i.e., the maximum possible for the order parameter squared amounts to one), as well as the correlation length $\xi$. These correlation lengths are again finite and range from $\xi(D=2) \approx 1.3$ to $\xi(D=5) \approx 8.4$. The correlation lengths for each $D$ are roughly a factor 2 larger than for the Heisenberg model. So it seems that the smaller number of Goldstone modes has a beneficial effect on the growth of the correlation lengths.

In Fig. 7, we present the energy per site $e$ as a function of $1 / \xi^{3}$ in the left-hand panel and $m^{2}$ as a function of $1 / \xi$ in the right-hand panel. This is the conjectured $\xi$ scaling form of Eqs. (22) and (23). We then fit the largest two $D$ values for the energy $e$ and all the $D$ values for $m^{2}$ and obtain the following iPEPS $\xi \rightarrow \infty$ estimates: $e_{X Y}^{(\mathrm{iPEPS})} \approx-0.548822$ [67] and $m_{X Y}^{2(\mathrm{PPEPS})}=0.7567(11)$. These values compare well with the QMC results of Ref. [68], $e_{X Y}^{(\mathrm{QMC})}=$ $-0.548824(2)$ and $m_{X Y}^{2(\mathrm{QMC})}=0.764(7)$. Since the QMC results are relatively antiquated, it is not inconceivable that the iPEPS results are actually more precise than the QMC results.

We are now also in a position to corroborate the $O(N)$ universality of the conjectured NLSM finite $\xi$ corrections.
Using the hydrodynamic parameters $v$ and $v / \rho_{s}$ from Ref. [68], the iPEPS amplitudes from Eqs. (25) and (26), and inserting $N=2$, we arrive at the NLSM predictions, which are shown by solid lines in both panels of Fig. 7. The nice agreement between the linear fits and the NLSM predictions for the slopes provides further support for the validity and therefore power of the field theoretically inspired finite- $\xi$ correction formulas.

\section{DISCUSSION AND INTERPRETATION}

After having studied the three different models we are confronted with the fact that in all cases the correlation length $\xi(D)$ was finite. While we have developed a powerful finite- $\xi$ scaling framework, where many observables, including energies and order parameters, can be analyzed and extrapolated to $\xi \rightarrow \infty$, we are still left in the dark both regarding the underlying origin of the finite $\xi(D)$ in the first place and regarding the functional dependence of $\xi$ on $D$ for a given model or universality class.

While we do not yet have compelling answers to both questions, we can at least try to shed as much light as possible based on our numerical data. In Fig. 8, we have assembled the correlation lengths observed in the critical transverse-field Ising model, which is described by one of the simplest nontrivial $3 d$ CFTs, together with two instances of $O(N)$ continuous symmetry-breaking phenomena with $N=3$ for the $S=1 / 2$ antiferromagnetic Heisenberg model and $N=2$ for the $S=1 / 2 X Y$ model. In the inset we also show the $D \geq 2$ data in a log-log plot, yielding some rough estimates for a putative power-law relation $\xi(D) \sim D^{\kappa}$ (it is not clear that such a law holds) [69]. With the two points for the critical transverse-field Ising model, we obtain $\kappa_{3 \mathrm{D} \text { Ising } \mathrm{CFT}} \approx 5$, while both 


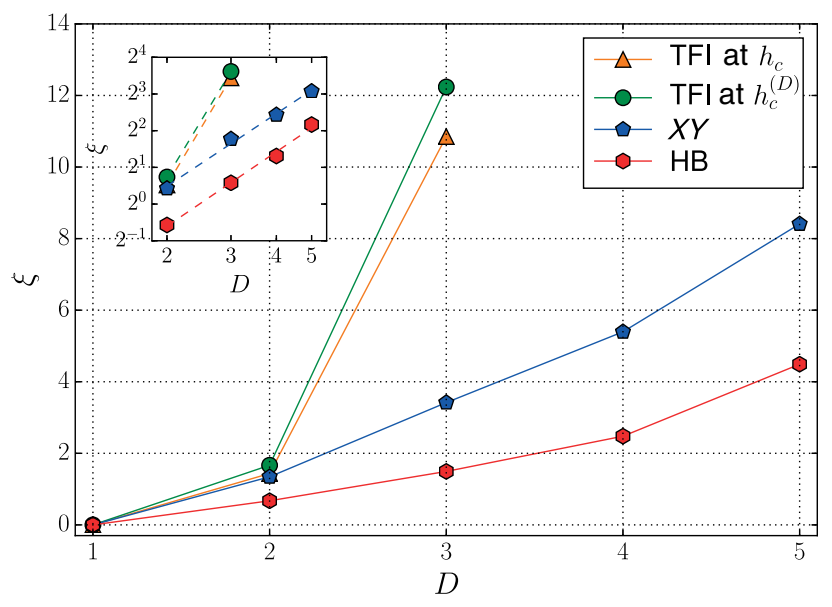

FIG. 8. Overview of the finite- $D$ iPEPS correlation lengths $\xi$ observed in the critical transverse-field Ising model (TFI) and the continuous symmetry-breaking $X Y$ and Heisenberg models. The inset displays the same data on logarithmic scales.

continuous symmetry-breaking cases seem to share the same $\kappa_{\mathrm{NLSM}} \approx 2$. The latter two cases however differ by a factor 2 in the prefactor, which incidentally is also the inverse ratio of the number of Goldstone modes. This could mean that the $X Y$ model has twice as large a correlation length as the Heisenberg model because it only has half the number of Goldstone modes. It would be interesting to explore whether the known additive logarithmic correction to the entanglement area law in continuous symmetrybreaking states [70] might be at the origin of this behavior. There the prefactor of the log contribution is proportional to the number of Goldstone modes. This would also explain why the speculative values of $\kappa$ are so different between the 3D Ising CFT and the symmetry-breaking cases. In terms of their low-energy degrees of freedom, the 3D Ising CFT and the $X Y$ model both contain a single real scalar field each-an interacting field in the Ising CFT case and a massless free field in the $X Y$ case. One could thus have expected the values of $\kappa$ to be roughly similar. The crucial difference therefore seems to come from the broken continuous symmetry in the $X Y$ model, which is absent in the Ising CFT.

The most pressing question remains, however, as to why we only find finite correlation lengths in the variationally optimized iPEPS wave functions for massless Lorentzinvariant $(2+1) d$ scenarios. We believe that our observations are actually the generic result, and that the previously known examples of iPEPS states with algebraic correlations are fine-tuned and nongeneric. As shown as an illustration in the left-hand panel of Fig. 9, we think of our $(2+1) d$ iPEPS states as wave functions whose correlation functions are represented by a path integral with a finite $\xi_{\tau}(D)$ extent in the (real or imaginary) time direction. The Lorentz invariance (or Euclidian invariance after a Wick rotation) of the fixed point we try to
$(2+1) d \mathrm{CFT} / \mathrm{QFT}$

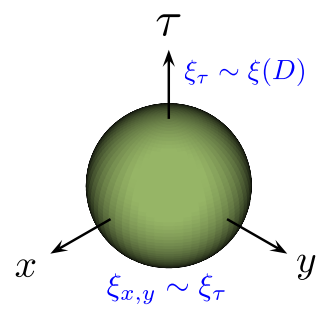

$(2+0) d$ Rokhsar-Kivelson

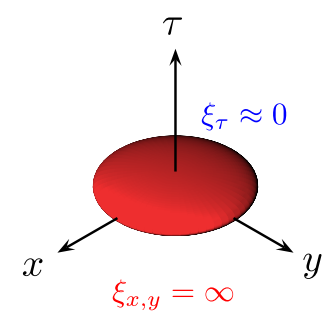

FIG. 9. Illustration of the space-time volume sampled in a $(2+1) d$ iPEPS for CFTs or quantum field theories (QFTs) versus the $(2+0) d$ Rokhsar-Kivelson-type iPEPS wave functions.

approximate then forces the spatial correlation lengths to be finite as well. The well-known iPEPS states with algebraic spatial correlations at finite $D$ can actually be represented by a purely in-plane path integral, where the temporal extent $\xi_{\tau}$ is basically zero (right-hand panel). This is certainly true for the $2 d$ classical partition function Ising PEPS [21], quantum dimer Rokhsar-Kivelson states [18,71], and certain quantum Lifshitz theories [72,73], where there is a built-in space-time asymmetry. Some further evidence supporting this picture might be obtained from the entanglement entropies $S_{\mathrm{bMPS}}$ of the boundary MPS resulting from the contractions of the iPEPS, as it seems plausible that this entanglement entropy is amplified if the correlation volume extends into the $\tau$ direction. The data shown in Fig. 10 can indeed be interpreted that the entanglement entropy grows more rapidly with the correlation length $\xi$ in the genuine $3 d$ space-time cases, compared to the quantitatively well-understood logarithmic scaling of the $(2+0) d$ wave functions, here exemplified by the Ising PEPS at various temperatures [74]. It would be interesting to study these boundary MPS entropies more systematically, as they roughly dictate how large the boundary $\chi$ value in the contraction schemes has to be.

Coming back to the reason for the finite $\xi_{\tau}(D)$ in the first place: We speculate that finding the dominant eigenvector of a plane-to-plane transfer operator along the temporal direction combined with a projection to a finite- $D$ iPEPS leads invariably to a finite correlation length in the temporal direction. This view is also supported by a more formal argument stating that an entropic area law does not automatically imply an efficient iPEPS representation [75].

To a first approximation we understand the finite- $D$ iPEPS to mimic a ground-state wave function of the field theoretical fixed point perturbed by a certain amount of the most relevant allowed perturbation given the symmetry constraints imposed on the iPEPS. Such states are driven away from the gapless situation, leading to a finite correlation length $\xi(D)$. In the examples studied here this always corresponds to a perturbation by coupling to the magnetic order parameter. Imposing symmetry constraints on the tensor might change the nature of the allowed relevant perturbation, and could also affect the values of 

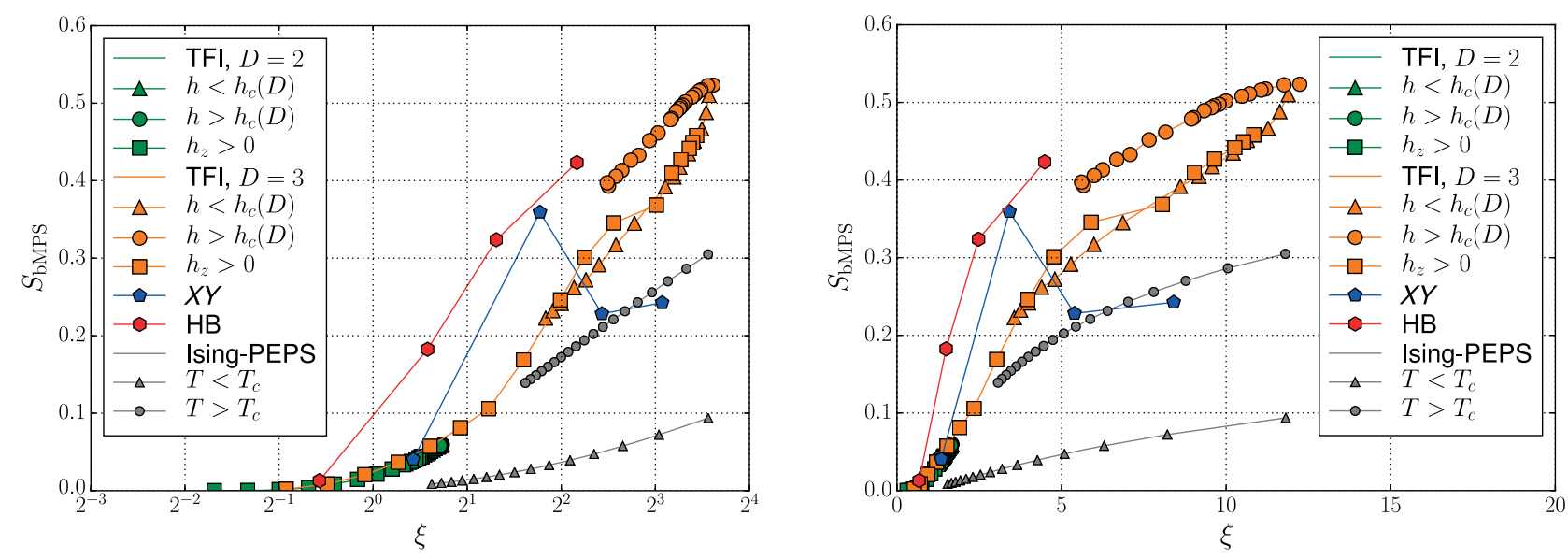

FIG. 10. Half-chain von Neumann entropy $S_{\text {bMPS }}$ of the boundary MPS resulting from the contraction of the iPEPS tensors, plotted as a function of $\log \xi$ (left-hand panel) and $\xi$ (right-hand panel). We display all available data sets. The transverse-field Ising model data are organized according to $D$ and the parameter cut, while the Heisenberg and $X Y$ model connect all available $D$ values. For comparison we also show entanglement entropies for the $(2+0) d$ Ising PEPS evaluated at different temperatures.

some of the finite- $\xi$ correction amplitudes introduced in this work.

\section{CONCLUSIONS}

In this paper, we introduce a powerful finite correlation length scaling framework for the analysis of finite- $D$ iPEPS which have been variationally optimized for Lorentz-invariant $(2+1) d$ quantum critical or continuous symmetry-breaking Hamiltonians. This framework is important (i) to understand the mild limitations of the finite- $D$ iPEPS manifold in representing Lorentz-invariant, gapless many-body quantum states and (ii) to put forward a practical scheme in which the finite correlation length $\xi(D)$ and field theory inspired formulas can be employed to extrapolate the data to infinite correlation length, i.e., to the thermodynamic limit. We show that some of the amplitudes entering the equations have a field theoretical interpretation and are therefore universal to some degree. We demonstrate the power of the method for the energy convergence in all three considered models, and for order parameter extrapolations for the continuous symmetrybreaking models, where the previously employed $1 / D$ extrapolation schemes performed poorly. We believe that another advantage of this approach is also that the variational quality of an iPEPS tensor and the resulting correlation length (and other observables) seem to go hand in hand in the vicinity of a local optimum, in such a way that different data points still lie on the same finite $\xi$ extrapolation curve.

We also carefully analyze the critical behavior of the transverse-field Ising model in the vicinity of its critical point by measurements of local observables as a function of the two magnetic fields, and we were able to obtain critical exponents within a few percent of the most recent conformal bootstrap results already at bond dimension $D=3$.
The finite correlation length scaling framework opens the way for further exploration of quantum matter with an (expected) Lorentz-invariant, massless low-energy description. Within the quantum critical or CFT related questions one could explore the Wilson-Fisher $O(N)$ fixed points beyond the $N=1$ case studied here, Gross-Neveu-Yukawa universality classes arising in interacting Dirac fermion systems, or $\mathrm{QED}_{3}$ related behavior of gapless quantum spin liquids or deconfined criticality. In the context of continuous symmetry breaking, various superfluid and superconducting phases in bosonic and fermionic systems should be described by the $O(2)$ symmetry-breaking results established in this work. Further directions are noncollinear magnetic order or $\mathrm{SU}(N)$ continuous symmetry breaking. Ultimately one should also explore the occurrence of finite correlation lengths and their scaling in interacting systems with a Fermi surface.

On the fundamental level it will be important to develop an understanding of the $\xi(D)$ relation, that we started to explore here. Our preliminary results indicate a $\xi \sim D^{\kappa}$ scaling with values of $\kappa$ which could be universal. In the $(1+1) d$ iMPS context, the values of $\kappa$ depend only on the central charge $c$ [8]. It would be interesting to understand in $(2+1) d$ whether $\kappa$ also depends only on some universal data, such as in the $F$ theorem [76,77].

\section{ACKNOWLEDGMENTS}

We acknowledge discussions with P. Corboz, J. Haegeman, G. Misguich, L. Tagliacozzo, L. Vanderstraeten, F. Verstraete, and G. Vidal. We thank P. Corboz for bringing Ref. [39] to our attention. We acknowledge support by the Austrian Science Fund FWF within the DK-ALM (W1259-N27) and the Spezialforschungsbereich FoQus (F-4018). The computational results presented have been achieved in part using the HPC infrastructure LEO of the University of Innsbruck. The 

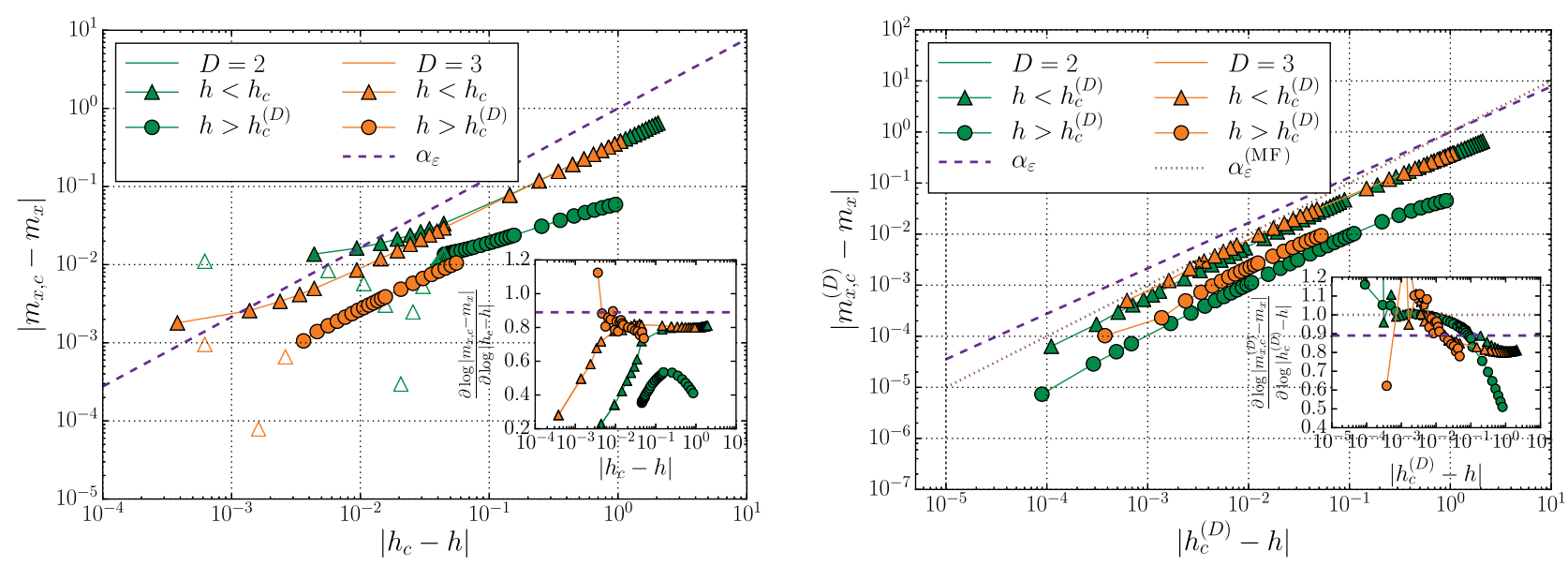

FIG. 11. Analysis of the critical behavior of the $m_{x}$ magnetizations in the transverse-field Ising model in the vicinity of the critical point at $h=h_{c}, h_{z}=0$ as a function of the transverse field. The left-hand panel shows $\left|m_{x, c}-m_{x}\right|$ as a function of $\left|h_{c}-h\right|$ and the righthand panel $\left|m_{x, c}^{(D)}-m_{x}\right|$ as a function of $\left|h_{c}^{(D)}-h\right|$. In the insets numerical derivatives are presented (where the hollow symbols of the left-hand panel are excluded as they cause divergences in the derivative). The inset of the right-hand panel shows mean-field behavior of the $D=2$ data at small $\left|h_{c}^{(D)}-h\right|$ while the $D=3$ data are too noisy to allow firm conclusions. The expected exponents are tabulated in Table I.

computational results presented have been achieved in part using the Vienna Scientific Cluster (VSC).

Note added.-Similar results have been reported by Corboz et al. [78].

\section{APPENDIX A: FURTHER RESULTS ON THE TRANSVERSE-FIELD ISING MODEL}

In this appendix, the critical behavior of the $m_{x}$ magnetizations as a function of the transverse field $h$ in the transverse-field Ising model in the vicinity of the critical point at $h=h_{c}, h_{z}=0$ is presented. In the left-hand panel of Fig. 11 we plot $\left|m_{x, c}-m_{x}\right|$ as a function of $\left|h_{c}-h\right|$ on a log-log scale together with a straight line showing the expected slope $\alpha_{\varepsilon}$ as a guide to the eye. In the corresponding inset we numerically calculate the derivative (excluding the hollow symbols as they cause divergences in the derivative). The data show convergence towards the

TABLE II. Best variational energies.

\begin{tabular}{ccc}
\hline \hline Model & $D$ & Energy $/ J$ \\
\hline \multirow{2}{*}{ TFI at $h_{c}$} & 2 & -3.233573421 \\
& 3 & -3.234260711 \\
$S=1 / 2 \mathrm{HB}$ & 2 & -0.660231093 \\
& 3 & -0.667974240 \\
& 4 & -0.669083757 \\
$S=1 / 2 X Y$ & 5 & -0.669378064 \\
& 2 & -0.547658559 \\
& 3 & -0.548706183 \\
& 4 & -0.548810284 \\
\hline \hline
\end{tabular}

expected value for the critical exponent $\alpha_{\varepsilon}$ from $D=2$ to $D=3$, although the noise on this data is relatively large compared to the data presented in Fig. 3. This might be due to the uncertainty in the estimation of $m_{x, c}$.

In the right-hand panel of Fig. 11, $\left|m_{x, c}^{(D)}-m_{x}\right|$ is presented as a function of $\left|h_{c}^{(D)}-h\right|$ on a log-log scale, and the inset contains the corresponding numerical derivatives. The $D=2$ data clearly show mean-field behavior at small $\left|h_{c}^{(D)}-h\right|$, but the derivatives for $D=3$ might already start to form an intermediate plateau. We expect data for $D>3$ to give a better understanding of how this plateau is formed.

\section{APPENDIX B: VARIATIONAL IPEPS ENERGIES}

For reference, we provide in Table II our best variational iPEPS energies for the three models considered in this paper. The transverse-field Ising model is at its critical point, $h=h_{c}, h_{z}=0$.

[1] S. R. White, Density Matrix Formulation for Quantum Renormalization Groups, Phys. Rev. Lett. 69, 2863 (1992).

[2] U. Schollwöck, The Density-Matrix Renormalization Group, Rev. Mod. Phys. 77, 259 (2005).

[3] G. Vidal, Classical Simulation of Infinite-Size Quantum Lattice Systems in One Spatial Dimension, Phys. Rev. Lett. 98, 070201 (2007).

[4] I. P. McCulloch, Infinite Size Density Matrix Renormalization Group, Revisited, arXiv:0804.2509.

[5] U. Schollwöck, The Density-Matrix Renormalization Group in the Age of Matrix Product States, Ann. Phys. (Amsterdam) 326, 96 (2011). 
[6] G. Vidal, Class of Quantum Many-Body States That Can Be Efficiently Simulated, Phys. Rev. Lett. 101, 110501 (2008).

[7] L. Tagliacozzo, T. R. de Oliveira, S. Iblisdir, and J. I. Latorre, Scaling of Entanglement Support for Matrix Product States, Phys. Rev. B 78, 024410 (2008).

[8] F. Pollmann, S. Mukerjee, A. M. Turner, and J. E. Moore, Theory of Finite-Entanglement Scaling at One-Dimensional Quantum Critical Points, Phys. Rev. Lett. 102, 255701 (2009).

[9] V. Stojevic, J. Haegeman, I. P. McCulloch, L. Tagliacozzo, and F. Verstraete, Conformal Data from Finite Entanglement Scaling, Phys. Rev. B 91, 035120 (2015).

[10] R. N. C. Pfeifer, G. Evenbly, and G. Vidal, Entanglement Renormalization, Scale Invariance, and Quantum Criticality, Phys. Rev. A 79, 040301 (2009).

[11] F. Verstraete and J. I. Cirac, Renormalization Algorithms for Quantum-Many Body Systems in Two and Higher Dimensions, arXiv:cond-mat/0407066.

[12] P. Corboz, S. R. White, G. Vidal, and M. Troyer, Stripes in the Two-Dimensional $t-J$ Model with Infinite Projected Entangled-Pair States, Phys. Rev. B 84, 041108 (2011).

[13] P. Corboz, M. Lajkó, A. M. Läuchli, K. Penc, and F. Mila, Spin-Orbital Quantum Liquid on the Honeycomb Lattice, Phys. Rev. X 2, 041013 (2012).

[14] D. Poilblanc, N. Schuch, D. Pérez-García, and J. I. Cirac, Topological and Entanglement Properties of Resonating Valence Bond Wave Functions, Phys. Rev. B 86, 014404 (2012).

[15] P. Corboz and F. Mila, Crystals of Bound States in the Magnetization Plateaus of the Shastry-Sutherland Model, Phys. Rev. Lett. 112, 147203 (2014).

[16] T. Picot, M. Ziegler, R. Orús, and D. Poilblanc, Spin-S Kagome Quantum Antiferromagnets in a Field with Tensor Networks, Phys. Rev. B 93, 060407 (2016).

[17] B.-X. Zheng, C.-M. Chung, P. Corboz, G. Ehlers, M.-P. Qin, R. M. Noack, H. Shi, S. R. White, S. Zhang, and G. K.-L. Chan, Stripe Order in the Underdoped Region of the TwoDimensional Hubbard Model, Science 358, 1155 (2017).

[18] N. Schuch, D. Poilblanc, J. I. Cirac, and D. Pérez-García, Resonating Valence Bond States in the PEPS Formalism, Phys. Rev. B 86, 115108 (2012).

[19] N. Schuch, D. Poilblanc, J. I. Cirac, and D. Pérez-García, Topological Order in the Projected Entangled-Pair States Formalism: Transfer Operator and Boundary Hamiltonians, Phys. Rev. Lett. 111, 090501 (2013).

[20] J. Eisert, M. Cramer, and M. B. Plenio, Colloquium: Area Laws for the Entanglement Entropy, Rev. Mod. Phys. 82, 277 (2010).

[21] F. Verstraete, M. M. Wolf, D. Perez-Garcia, and J. I. Cirac, Criticality, the Area Law, and the Computational Power of Projected Entangled Pair States, Phys. Rev. Lett. 96, 220601 (2006).

[22] J. Jordan, R. Orús, G. Vidal, F. Verstraete, and J. I. Cirac, Classical Simulation of Infinite-Size Quantum Lattice Systems in Two Spatial Dimensions, Phys. Rev. Lett. 101, 250602 (2008).

[23] R. Orús and G. Vidal, Simulation of Two-Dimensional Quantum Systems on an Infinite Lattice Revisited: Corner Transfer Matrix for Tensor Contraction, Phys. Rev. B 80, 094403 (2009).
[24] T. Nishino and K. Okunishi, Corner Transfer Matrix Renormalization Group Method, J. Phys. Soc. Jpn. 65, 891 (1996).

[25] M. Levin and C. P. Nave, Tensor Renormalization Group Approach to Two-Dimensional Classical Lattice Models, Phys. Rev. Lett. 99, 120601 (2007).

[26] Z.-C. Gu, M. Levin, and X.-G. Wen, Tensor-Entanglement Renormalization Group Approach as a Unified Method for Symmetry Breaking and Topological Phase Transitions, Phys. Rev. B 78, 205116 (2008).

[27] Z. Y. Xie, H. C. Jiang, Q. N. Chen, Z. Y. Weng, and T. Xiang, Second Renormalization of Tensor-Network States, Phys. Rev. Lett. 103, 160601 (2009).

[28] Z. Y. Xie, J. Chen, M. P. Qin, J. W. Zhu, L. P. Yang, and T. Xiang, Coarse-Graining Renormalization by Higher-Order Singular Value Decomposition, Phys. Rev. B 86, 045139 (2012).

[29] G. Evenbly and G. Vidal, Tensor Network Renormalization, Phys. Rev. Lett. 115, 180405 (2015).

[30] M. Fishman, L. Vanderstraeten, V. Zauner-Stauber, J. Haegeman, and F. Verstraete, Faster Methods for Contracting Infinite 2D Tensor Networks, arXiv:1711.05881.

[31] P. Corboz, T. M. Rice, and M. Troyer, Competing States in the t-J Model: Uniform d-Wave State versus Stripe state, Phys. Rev. Lett. 113, 046402 (2014).

[32] P. Corboz, Variational Optimization with Infinite Projected Entangled-Pair States, Phys. Rev. B 94, 035133 (2016).

[33] L. Vanderstraeten, J. Haegeman, P. Corboz, and F. Verstraete, Gradient Methods for Variational Optimization of Projected Entangled-Pair States, Phys. Rev. B 94, 155123 (2016).

[34] C. G. Broyden, The Convergence of a Class of Double-Rank Minimization Algorithms 1. General Considerations, IMA J. Appl. Math. 6, 76 (1970).

[35] R. Fletcher, A New Approach to Variable Metric Algorithms, Computer Journal (UK) 13, 317 (1970).

[36] D. Goldfarb, A Family of Variable-Metric Methods Derived by Variational Means, Math. Comput. 24, 23 (1970).

[37] D. F. Shanno, Conditioning of Quasi-Newton Methods for Function Minimization, Math. Comput. 24, 647 (1970).

[38] M. Rader and A. M. Läuchli (unpublished).

[39] M. M. Rams, P. Czarnik, and L. Cincio, Precise Extrapolation of the Correlation Function Asymptotics in Uniform Tensor Network States with Application to the Bose-Hubbard and XXZ Models, arXiv:1801.08554.

[40] H. W. J. Blöte and Y. Deng, Cluster Monte Carlo Simulation of the Transverse Ising Model, Phys. Rev. E 66, 066110 (2002).

[41] F. Kos, D. Poland, D. Simmons-Duffin, and A. Vichi, Precision Islands in the Ising and $O(N)$ Models, J. High Energy Phys. 08 (2016) 36.

[42] Z.-C. Gu, M. Levin, and X.-G. Wen, Tensor-Entanglement Renormalization Group Approach as a Unified Method for Symmetry Breaking and Topological Phase Transitions, Phys. Rev. B 78, 205116 (2008).

[43] R. Orús, Exploring Corner Transfer Matrices and Corner Tensors for the Classical Simulation of Quantum Lattice Systems, Phys. Rev. B 85, 205117 (2012).

[44] C. Liu, L. Wang, A. W. Sandvik, Y.-C. Su, and Y.-J. Kao, Symmetry Breaking and Criticality in Tensor-Product States, Phys. Rev. B 82, 060410 (2010). 
[45] M. Lubasch, J. I. Cirac, and M.-C. Bañuls, Algorithms for Finite Projected Entangled Pair States, Phys. Rev. B 90, 064425 (2014).

[46] H. N. Phien, I. P. McCulloch, and G. Vidal, Fast Convergence of Imaginary Time Evolution Tensor Network Algorithms by Recycling the Environment, Phys. Rev. B 91, 115137 (2015).

[47] H. N. Phien, J. A. Bengua, H. D. Tuan, P. Corboz, and R. Orús, Infinite Projected Entangled Pair States Algorithm Improved: Fast Full Update and Gauge Fixing, Phys. Rev. B 92, 035142 (2015).

[48] T. Nishino, Y. Hieida, K. Okunishi, N. Maeshima, Y. Akutsu, and A. Gendiar, Two-Dimensional Tensor Product Variational Formulation, Prog. Theor. Phys. 105, 409 (2001).

[49] A. Gendiar, N. Maeshima, and T. Nishino, Stable Optimization of a Tensor Product Variational State, Prog. Theor. Phys. 110, 691 (2003).

[50] Since the energy optimization of states with such a large correlation length is computationally quite demanding, there is a small uncertainty in the value of $\xi_{\max }^{(D=3)}$.

[51] M. Schuler, S. Whitsitt, L.-P. Henry, S. Sachdev, and A. M. Läuchli, Universal Signatures of Quantum Critical Points from Finite-Size Torus Spectra: A Window into the Operator Content of Higher-Dimensional Conformal Field Theories, Phys. Rev. Lett. 117, 210401 (2016).

[52] A square torus is a quadratic system with periodic boundary conditions in both orthogonal spatial directions. For details on the definition of the modular parameter, see Ref. [51].

[53] C. J. Hamer, Finite-Size Scaling in the Transverse Ising Model on a Square Lattice, J. Phys. A 33, 6683 (2000).

[54] We actually believe that the data in Fig. 5 ultimately organizes in several families with different Casimir amplitudes, but all sharing a $1 / \xi^{3}$ scaling.

[55] S. Chakravarty, B. I. Halperin, and D. R. Nelson, TwoDimensional Quantum Heisenberg Antiferromagnet at Low Temperatures, Phys. Rev. B 39, 2344 (1989).

[56] A. W. Sandvik, Finite-Size Scaling of the Ground-State Parameters of the Two-Dimensional Heisenberg Model, Phys. Rev. B 56, 11678 (1997).

[57] H. Neuberger and T. Ziman, Finite-Size Effects in Heisenberg Antiferromagnets, Phys. Rev. B 39, 2608 (1989).

[58] D. S. Fisher, Universality, Low-Temperature Properties, and Finite-Size Scaling in Quantum Antiferromagnets, Phys. Rev. B 39, 11783 (1989).

[59] P. Hasenfratz and H. Leutwyler, Goldstone Boson Related Finite Size Effects in Field Theory and Critical Phenomena with $O(N)$ Symmetry, Nucl. Phys. B343, 241 (1990).

[60] P. Hasenfratz and F. Niedermayer, Finite Size and Temperature Effects in the AF Heisenberg Model, Z. Phys. B 92, 91 (1993).

[61] B. Bauer, G. Vidal, and M. Troyer, Assessing the Accuracy of Projected Entangled-Pair States on Infinite Lattices, J. Stat. Mech. (2009) P09006.

[62] B. Bauer, P. Corboz, R. Orús, and M. Troyer, Implementing Global Abelian Symmetries in Projected Entangled-Pair State Algorithms, Phys. Rev. B 83, 125106 (2011).
[63] P. Corboz, Improved Energy Extrapolation with Infinite Projected Entangled-Pair States Applied to the TwoDimensional Hubbard Model, Phys. Rev. B 93, 045116 (2016).

[64] A. W. Sandvik and H. G. Evertz, Loop Updates for Variational and Projector Quantum Monte Carlo Simulations in the Valence-Bond Basis, Phys. Rev. B 82, 024407 (2010).

[65] F.-J. Jiang and U.-J. Wiese, High-Precision Determination of Low-Energy Effective Parameters for a Two-Dimensional Heisenberg Quantum Antiferromagnet, Phys. Rev. B 83, 155120 (2011).

[66] J. Jordan, R. Orús, and G. Vidal, Numerical Study of the Hard-Core Bose-Hubbard Model on an Infinite Square Lattice, Phys. Rev. B 79, 174515 (2009).

[67] The extrapolation with $D=4$ and $D=5$ alone gives an energy density of $e_{X Y}^{(\mathrm{iPEPS})} \approx-0.548822$. However, from this extrapolation we cannot extract a numerical error.

[68] A. W. Sandvik and C. J. Hamer, Ground-State Parameters, Finite-Size Scaling, and Low-Temperature Properties of the Two-Dimensional $S=\frac{1}{2} X Y$ Model, Phys. Rev. B 60, 6588 (1999).

[69] R. Haghshenas and D. N. Sheng, U(1)-Symmetric Infinite Projected Entangled-Pair States Study of the Spin-1/2 Square $J_{1}-J_{2}$ Heisenberg Model, Phys. Rev. B 97, 174408 (2018).

[70] M. A. Metlitski and T. Grover, Entanglement Entropy of Systems with Spontaneously Broken Continuous Symmetry, arXiv:1112.5166.

[71] C. Castelnovo, C. Chamon, C. Mudry, and P. Pujol, From Quantum Mechanics to Classical Statistical Physics: Generalized Rokhsar-Kivelson Hamiltonians and the "Stochastic Matrix Form” Decomposition, Ann. Phys. (Amsterdam) 318, 316 (2005).

[72] S. V. Isakov, P. Fendley, A. W. W. Ludwig, S. Trebst, and M. Troyer, Dynamics At and Near Conformal Quantum Critical Points, Phys. Rev. B 83, 125114 (2011).

[73] B. Hsu and E. Fradkin, Dynamical Stability of the Quantum Lifshitz. Theory in 2+1 Dimensions, Phys. Rev. B 87, 085102 (2013).

[74] The $X Y$ model shows a somewhat irregular behavior, whose origin we do not understand.

[75] Y. Ge and J. Eisert, Area Laws and Efficient Descriptions of Quantum Many-Body States, New J. Phys. 18, 083026 (2016).

[76] H. Casini, M. Huerta, and R. C. Myers, Towards a Derivation of Holographic Entanglement Entropy, J. High Energy Phys. 05 (2011) 36.

[77] T. Grover, Entanglement Monotonicity and the Stability of Gauge Theories in Three Spacetime Dimensions, Phys. Rev. Lett. 112, 151601 (2014).

[78] P. Corboz, P. Czarnik, G. Kapteijns, and L. Tagliacozzo, following article, Finite Correlation Length Scaling with Infinite Projected Entangled-Pair States, Phys. Rev. X 8, 031031 (2018). 